\title{
NON-COMMUTATIVE IWASAWA THEORY FOR ELLIPTIC CURVES WITH MULTIPLICATIVE REDUCTION
}

\author{
by Daniel Delbourgo and Antonio Lei
}

\begin{abstract}
Let $E_{/ \mathbb{Q}}$ be a semistable elliptic curve, and $p \neq 2$ a prime of bad multiplicative reduction. For each Lie extension $\mathbb{Q}_{F T} / \mathbb{Q}$ with Galois group $G_{\infty} \cong \mathbb{Z}_{p} \rtimes \mathbb{Z}_{p}^{\times}$, we construct p-adic L-functions interpolating Artin twists of the Hasse-Weil L-series of the curve $E$. Through the use of congruences, we next prove a formula for the analytic $\lambda$-invariant over the false Tate tower, analogous to Chern-Yang Lee's results on its algebraic counterpart. If one assumes the Pontryagin dual of the Selmer group belongs to the $\mathfrak{M}_{\mathcal{H}}\left(G_{\infty}\right)$-category, the leading terms of its associated Akashi series can then be computed, allowing us to formulate a non-commutative Iwasawa Main Conjecture in the multiplicative setting.
\end{abstract}

\section{Introduction}

Fix a prime number $p \neq 2$, and let $\Delta>1$ denote a $p$-power free integer coprime to $p$. For each integer $n \geq 0$, we set $K_{n}=\mathbb{Q}\left(\mu_{p^{n}}\right)$ and write $F_{n}=\mathbb{Q}\left(\mu_{p^{n}}\right)^{+}$for the maximal real subfield. We construct a $p$-adic Lie extension of $\mathbb{Q}$ by taking a union of the fields $L_{n}=\mathbb{Q}\left(\mu_{p^{n}}, \sqrt[p^{n}]{\Delta}\right)$, so that $\mathbb{Q}_{F T}:=\bigcup_{n \geq 1} L_{n}$ has Galois group

$$
\operatorname{Gal}\left(\mathbb{Q}_{F T} / \mathbb{Q}\right) \cong\left(\begin{array}{cc}
\mathbb{Z}_{p}^{\times} & \mathbb{Z}_{p} \\
0 & 1
\end{array}\right) \triangleleft \mathrm{GL}_{2}\left(\mathbb{Z}_{p}\right)
$$

which is a semi-direct product. In terms of a tower diagram for the various field extensions:

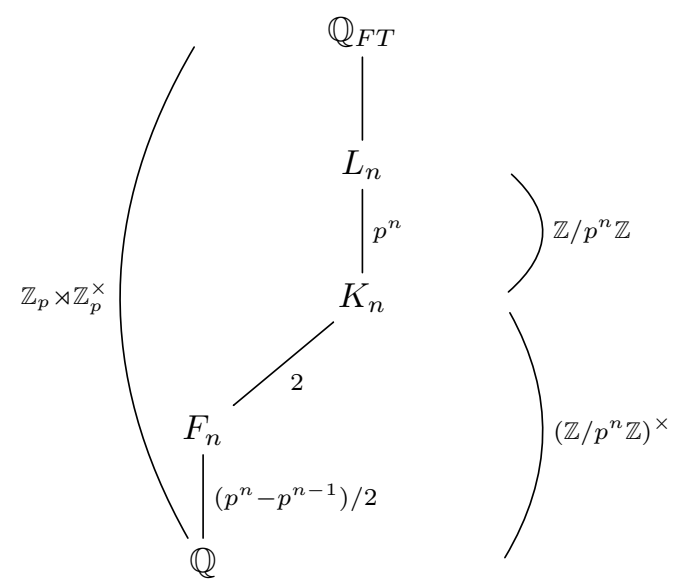

2010 Mathematics Subject Classification: 11R23 (primary); 11G40, 19B28

Department of Mathematics, University of Waikato, Hamilton 3240, New Zealand

Département de mathématiques et de statistique, Université Laval, Québec QC, Canada G1V 0A6. 
It is proved in $[12]$ that $G_{\infty}:=\operatorname{Gal}\left(\mathbb{Q}_{F T} / \mathbb{Q}\right)$ has a unique self-dual representation of dimension $p^{k}-p^{k-1}$ for each $k \geq 1$, which is of the form

$$
\rho_{k}=\rho_{k, \mathbb{Q}}=\operatorname{Ind}_{K_{k}}^{\mathbb{Q}}\left(\chi_{\rho_{k}}\right)
$$

for any character $\chi_{\rho_{k}}: \operatorname{Gal}\left(L_{k} / K_{k}\right) \rightarrow \mu_{p^{k}}$ (e.g. the map sending $\sigma \mapsto \frac{\sigma(\sqrt[p]{\Delta})}{p^{k} \sqrt{\Delta}}$ will do). Setting $\rho_{0, \mathbb{Q}}=\mathbf{1}$, then every irreducible representation of $\operatorname{Gal}\left(\mathbb{Q}_{F T} / \mathbb{Q}\right)$ is isomorphic to $\rho_{k, \mathbb{Q}} \otimes \psi$ for some $k \geq 0$, and some finite order character $\psi: \operatorname{Gal}\left(\mathbb{Q}\left(\mu_{p^{\infty}}\right) / \mathbb{Q}\right) \rightarrow \mathbb{C}^{\times}$.

Let $E$ be a semistable elliptic curve defined over the rationals; in particular, it is modular by the work of Wiles. We denote by $f_{E}=\sum_{n=1}^{\infty} a_{n}(E) \exp (2 \pi i n z)$ the newform of weight two and conductor $N_{E}$ associated to $E_{/ \mathbb{Q}}$, so $N_{E}$ is square-free as $E$ is semistable. In this article we shall assume that $\Delta$ is coprime to $N_{E}$, and throughout impose

Hypothesis(Mult): The elliptic curve $E$ has bad multiplicative reduction at $p$.

Recall that for an Artin representation $\rho$ over $F$, one can form the $\rho$-twisted $L$-function $L(E, \rho, s)$ by taking the Euler product

$$
L(E, \rho, s)=\prod_{v} \operatorname{det}\left(1-N_{F / \mathbb{Q}}(v)^{-s} \vartheta_{v} \mid\left(H_{l}^{1}(E) \otimes_{\overline{\mathbb{Q}}_{l}} \rho\right)^{I_{v}}\right)^{-1}
$$

where $\vartheta_{v}$ is a geometric Frobenius element for $v$, and $I_{v}$ the inertia group; this product converges to an analytic function on $\operatorname{Re}(s)>3 / 2$. We write $\Omega_{E}^{ \pm}$for the real/imaginary periods of a Néron differential associated to a minimal Weierstrass equation for $E / \mathbb{Q}$.

Theorem 1. Let $\mathfrak{p}$ and $\mathfrak{P}$ denote the primes of $F_{n}$ and $K_{n}$ lying above $p$ respectively, define $U^{(n)}:=\operatorname{ker}\left(\mathbb{Z}_{p}^{\times} \rightarrow\left(\mathbb{Z} / p^{n} \mathbb{Z}\right)^{\times}\right)$, and write $S$ for the set of places of $\mathcal{O}_{F_{n}}$ over $\Delta$. For every $n \geq 1$, there exists a unique element $\mathbf{L}_{p}\left(E, \rho_{n}\right) \in \mathbb{Z}_{p}\left[\left[U^{(n)}\right]\right] \otimes \mathbb{Q}$ satisfying

$$
\psi\left(\mathbf{L}_{p}\left(E, \rho_{n}\right)\right)=\frac{\epsilon_{F_{n}}\left(\rho_{n} \otimes \psi\right)_{\mathfrak{p}}}{a_{p}(E)^{f\left(\rho_{n} \otimes \psi, \mathfrak{p}\right)}} \times\left(1-a_{p}(E) \chi_{\rho_{n}}(\mathfrak{P}) \psi^{-1}(\mathfrak{p})\right) \times \frac{L_{S}\left(E, \rho_{n} \otimes \psi^{-1}, 1\right)}{\left(\Omega_{E}^{+} \Omega_{E}^{-}\right)^{\phi\left(p^{n}\right) / 2}}
$$

at all finite characters $\psi$ of $U^{(n)}$.

Here $\epsilon_{F_{n}}\left(\rho_{n} \otimes \psi\right)_{\mathfrak{p}}$ denotes the local $\epsilon$-factor at $\mathfrak{p}$. The $\epsilon$-factor depends on the choice of a local Haar measure and an additive character at $p$ (see [24] for details). We choose the Haar measure $\mathrm{d} x$ which gives $\mathbb{Z}_{p}$ measure 1 , and the additive character $\tau:\left(\mathbb{Q}_{p},+\right) \longrightarrow \mathbb{C}^{\times}$ given by $\tau\left(a p^{-m}\right)=\exp \left(2 \pi i a / p^{m}\right)$ with $a \in \mathbb{Z}_{p}$ (these are the choices used in [4]).

Analogous $p$-adic $L$-functions were constructed in $[2,7]$ for the good ordinary case, and we shall show that similar congruences to op. cit. hold in our setting, under the following condition.

Hypothesis $(\mu=0)$ : The analytic $\mu$-invariant of $\mathbf{L}_{p}\left(E, \rho_{n}\right)$ equals zero at each $n \geq 0$.

In particular the above condition immediately implies integrality of the $\rho_{n}$-twisted $p$-adic $L$-functions for $E$, and allows us to jettison some of the tedious technical assumptions made in $[7,8]$. 
We now choose $a_{n}=\mathbf{L}_{p}\left(E, \rho_{n}\right)$ for $n \geq 1$, and take $a_{0} \in \mathbb{Z}_{p}\left[\left[U^{(0)}\right]\right] \otimes \mathbb{Q}$ to be the Mazur-Tate-Teitelbaum $p$-adic $L$-function from [17], which exhibits $p-1$ branches in $\mathbb{Z}_{p}[[T]] \otimes \mathbb{Q}$. For integers $j>i \geq 0$, let $N_{i, j}: \mathbb{Z}_{p}\left[\left[U^{(i)}\right]\right] \rightarrow \mathbb{Z}_{p}\left[\left[U^{(j)}\right]\right]$ denote the norm map, and $\phi: \mathbb{Z}_{p}\left[\left[\mathbb{Z}_{p}^{\times}\right]\right] \rightarrow \mathbb{Z}_{p}\left[\left[\mathbb{Z}_{p}^{\times}\right]\right]$be the ring homomorphism induced by the $p$-power map on $\mathbb{Z}_{p}^{\times}$. In particular $N_{0,1}\left(a_{0}\right)$ is the product $\prod_{j=0}^{p-2} \mathbf{L}_{p}\left(E, \omega_{p}^{j}\right)$, where $\omega_{p}$ is the Teichmüller character modulo $p$.

Theorem 2. Under the Hypothesis $(\mu=0)$, for $n \geq 1$ there are "first layer" congruences

$$
a_{n} \equiv N_{0, n}\left(a_{0}\right) \quad \bmod p \mathbb{Z}_{p}\left[\left[U^{(n)}\right]\right]
$$

Coates et al [4] conjectured the existence of a non-abelian $p$-adic $L$-function when $E$ has good ordinary reduction at $p$ - we will now give an analogous conjecture in our setting. Let $\mathcal{H}=\operatorname{Gal}\left(\mathbb{Q}_{F T} / \mathbb{Q}^{\text {cyc }}\right) \cong \mathbb{F}_{p}^{\times} \ltimes \mathbb{Z}_{p}$ where $\mathbb{Q}^{\text {cyc }}$ means the cyclotomic $\mathbb{Z}_{p}$-extension of $\mathbb{Q}$. We denote by $\mathcal{S}$ the set

$$
\left\{x \in \mathbb{Z}_{p}\left[\left[G_{\infty}\right]\right]: \mathbb{Z}_{p}\left[\left[G_{\infty}\right]\right] / \mathbb{Z}_{p}\left[\left[G_{\infty}\right]\right] x \text { is a finitely generated } \mathbb{Z}_{p}[[\mathcal{H}]] \text {-module }\right\}
$$

and write $\mathbb{Z}_{p}\left[\left[G_{\infty}\right]\right]_{\mathcal{S}^{*}}$ for the localisation of $\mathbb{Z}_{p}\left[\left[G_{\infty}\right]\right]$ at its $p$-saturation $\mathcal{S}^{*}=\bigcup_{n \geq 0} p^{n} \mathcal{S}$. One predicts there exists a special element

$$
\mathbf{L}_{p}^{\text {anal }}\left(E / \mathbb{Q}_{F T}\right) \in \mathrm{K}_{1}\left(\mathbb{Z}_{p}\left[\left[G_{\infty}\right]\right]_{\mathcal{S}^{*}}\right)
$$

whose evaluation at Artin representations $\rho: G_{\mathbb{Q}} \rightarrow G_{\infty} \rightarrow \mathrm{GL}(V)$ essentially yield the $\rho$-twisted $L$-values $L(E, \rho, 1)$, up to some simple $p$-adic interpolation factors.

In [15] Kato reduced the question of existence for $\mathbf{L}_{p}^{\text {anal }}$ into a sequence of congruence relations, amongst the abelian $p$-adic $L$-functions $\left\{a_{n}\right\}_{n \geq 0}$ interpolating $E$ over the false Tate curve extension. More precisely, he constructed a theta-mapping

$$
\Theta_{G_{\infty}, \mathcal{S}^{*}}: \mathrm{K}_{1}\left(\mathbb{Z}_{p}\left[\left[G_{\infty}\right]\right]_{\mathcal{S}^{*}}\right) \stackrel{\left(\rho_{n}\right)_{*}}{\longrightarrow} \prod_{n \geq 0} \operatorname{Quot}\left(\mathbb{Z}_{p}\left[\left[U^{(n)}\right]\right]\right)^{\times}
$$

and determined the image of $\Theta_{G_{\infty}, \mathcal{S}^{*}}$ entirely through the use of $p$-power congruences. Using the same arguments in $[7, \S 3.3]$, we deduce from Theorem 2 the following

Corollary 3. Under the same Hypothesis $(\mu=0)$, the non-abelian congruences

$$
\prod_{1 \leq i \leq n} N_{i, n}\left(\frac{a_{i}}{N_{0, i}\left(a_{0}\right)} \cdot \frac{\phi \circ N_{0, i-1}\left(a_{0}\right)}{\phi\left(a_{i-1}\right)}\right)^{p^{i}} \equiv 1 \quad \bmod p^{n+1} \mathbb{Z}_{p}\left[\left[U^{(n)}\right]\right]_{(p)} \quad \text { hold for all } n \in \mathbb{N} .
$$

These congruences are necessary conditions to imply existence of $\mathbf{L}_{p}^{\text {anal }}\left(E / \mathbb{Q}_{F T}\right)$. If the congruences could be strengthened from $p^{n+1}$ to $p^{2 n}$, then these stronger versions would also be sufficient to fully establish existence, using [15, Example 8.12].

Remarks: (a) Theorem 1 allows us to define $p$-adic $L$-functions for a number field $F \subset \mathbb{Q}_{F T}$ by taking the product

$$
\mathbf{L}_{p}(E / F, T):=\prod_{\rho} \mathbf{L}_{p}(E, \rho, T)
$$

over irreducible sub-representations of $\operatorname{Ind}_{F}^{\mathbb{Q}} \mathbf{1}$, counted with multiplicity. We regard the $p$-adic $L$-functions above as elements of $\mathbb{Z}_{p}\left[\left[U^{(1)}\right]\right] \cong \mathbb{Z}_{p}[[T]]$ via the inclusion $U^{(n)} \hookrightarrow U^{(1)}$. 
(b) For example if $F / \mathbb{Q}$ is Galois, we have

$$
\mathbf{L}_{p}(E / F, T)=\prod_{\rho \in \operatorname{Irr}(\operatorname{Gal}(F / \mathbb{Q}))} \mathbf{L}_{p}(E, \rho, T)^{\operatorname{deg}(\rho)} .
$$

(c) If $F=\mathbb{Q}(\sqrt[p]{\Delta})$ for some $n \geq 1$, it is proved in $[12, \S 5.2]$ that $\operatorname{Ind}_{F}^{\mathbb{Q}} \mathbf{1} \cong \mathbf{1} \oplus \bigoplus_{j=1}^{n} \rho_{j}$ hence one obtains

$$
\mathbf{L}_{p}(E / F, T)=\mathbf{L}_{p}\left(E, \omega_{p}^{0}, T\right) \times \prod_{j=1}^{n} \mathbf{L}_{p}\left(E, \rho_{j}, T\right) .
$$

Let us define a non-negative integer $\delta:=\operatorname{ord}_{p}\left(\Delta^{p-1}-1\right)-1$, depending only on $p$ and $\Delta$.

Theorem 4. If $E$ has split multiplicative reduction at $p$, then

(i) $\mathbf{L}_{p}\left(E, \rho_{n}\right)$ has a trivial zero at $T=0$ if and only if $n \leq \delta$.

(ii) $\mathbf{L}_{p}\left(E / \mathbb{Q}\left(\sqrt[p^{n}]{\Delta}\right), T\right)$ has a trivial zero at $T=0$ of order $\geq \delta_{n}$, where $\delta_{n}$ denotes the number of primes in $\mathbb{Q}\left(\sqrt[p^{n}]{\Delta}\right)$ lying above $p$.

However if $E$ has non-split multiplicative reduction at $p$, there are no trivial zeros.

In the case $n \leq \delta$, a trivial zero formula computing the value of $\mathbf{L}_{p}^{\prime}\left(E, \rho_{n}\right)$ at $T=0$ should be possible if one employs a deformation theory argument along the lines of $[13,9,10]$. Given a number field $F \subset \mathbb{Q}_{F T}$, consider the integer

$$
r_{F}^{\dagger}(E):=\operatorname{order}_{T=0}\left(\mathbf{L}_{p}(E / F, T)\right)-\#\{\text { places } \nu \text { of } F \text { over } p\} \times \frac{1+a_{p}(E)}{2} .
$$

The term at the end is zero unless $E$ has split multiplicative reduction at $p$, in which case we need to offset the order of vanishing by each trivial zero contribution.

Conjecture 5. $\left(B S D_{p}\right) \quad r_{F}^{\dagger}(E)=\operatorname{dim}_{\mathbb{Q}}(E(F) \otimes \mathbb{Q})$.

For instance, assuming that the $p$-adic Birch and Swinnerton-Dyer Conjecture above holds over $F$, the quantity $r_{F}^{\dagger}(E)$ should correspond precisely to the Mordell-Weil rank of $E(F)$. In the following discussion, let $\lambda_{F}^{\text {an }}(E)$ be the number of zeros (counted with multiplicity) of the function $\mathbf{L}_{p}(E / F, T)$ on the open $p$-adic unit disk.

Theorem 6. (i) Under our Hypothesis( $\mu=0)$, for all integers $n \geq 1$

$$
r_{L_{n}}^{\dagger}(E) \leq p^{n} \times \lambda_{\mathbb{Q}\left(\mu_{p}\right)}^{\mathrm{an}}(E)-\left(\frac{1+a_{p}(E)}{2}\right) \times p^{\min (\delta, n)} .
$$

(ii) Moreover for the non-Galois extensions $F=\mathbb{Q}\left(\sqrt[p^{n}]{\Delta}\right)$, there are similar bounds

$$
r_{\mathbb{Q}\left(\sqrt[p^{n}]{\Delta}\right)}^{\dagger}(E) \leq\left(\frac{p^{n}-1}{p-1}\right) \times \lambda_{\mathbb{Q}\left(\mu_{p}\right)}^{\text {an }}(E)-\left(\frac{1+a_{p}(E)}{2}\right) \times \delta_{n}+\lambda_{\mathbb{Q}}^{\text {an }}(E) .
$$


Chern-Yang Lee has derived inequalities analogous to those above, with the quantities $r_{L_{n}}^{\dagger}(E)$ and $r_{\mathbb{Q}\left(\sqrt[p^{n}]{\Delta}\right)}^{\dagger}(E)$ replaced by the associated Selmer ranks over the number fields. In particular if $\delta=0$ and the generic parity is odd, it is shown in [16, Theorem 1.11] that

$$
\operatorname{corank}_{\mathbb{Z}_{p}} \operatorname{Sel}_{p}\left(E / L_{n}\right) \geq p^{n}-1+\operatorname{corank}_{\mathbb{Z}_{p}} \operatorname{Sel}_{p \infty}\left(E / \mathbb{Q}\left(\mu_{p}\right)\right)
$$

while for the non-Galois extensions,

$$
\operatorname{corank}_{\mathbb{Z}_{p}} \operatorname{Sel}_{p}(E / \mathbb{Q}(\sqrt[p n]{\Delta})) \geq n+\operatorname{corank}_{\mathbb{Z}_{p}} \operatorname{Sel}_{p^{\infty}}(E / \mathbb{Q}) .
$$

Note that Lee obtains lower bounds rather than upper bounds, as he is directly inputting parity information derived from a root number formula of V. Dokchitser [12, Theorem 1]. Both Lee's inequalities and our algebraic results below are dependent on various hypotheses concerning the structure of the Selmer group over $\mathbb{Q}_{F T}$, which we now describe.

Let $M=\operatorname{Hom}_{\text {cts }}\left(\operatorname{Sel}_{p \infty}\left(E / \mathbb{Q}_{F T}\right), \mathbb{Q} / \mathbb{Z}\right)$ denote the Pontryagin dual of the $p^{\infty}$-Selmer group for $E$ over the false Tate extension, and recall $\mathcal{H}$ was the Galois group $\operatorname{Gal}\left(\mathbb{Q}_{F T} / \mathbb{Q}^{\text {cyc }}\right)$. We assume throughout that $M$ belongs to the category $\mathfrak{M}_{\mathcal{H}}\left(G_{\infty}\right)$, so that $M / M_{p}$ is of finite-type over $\mathbb{Z}_{p}[[\mathcal{H}]]$. Since $G_{\infty}$ has no $p$-torsion, there is a surjective connecting map

$$
\partial_{G_{\infty}}: \mathrm{K}_{1}\left(\mathbb{Z}_{p}\left[\left[G_{\infty}\right]\right]_{\mathcal{S}^{*}}\right) \rightarrow \mathrm{K}_{0}\left(\mathfrak{M}_{\mathcal{H}}\left(G_{\infty}\right)\right)
$$

and write $\xi_{M}$ for a (characteristic) element in $\mathrm{K}_{1}\left(\mathbb{Z}_{p}\left[\left[G_{\infty}\right]\right]_{\mathcal{S}^{*}}\right)$ satisfying $\partial_{G_{\infty}}\left(\xi_{M}\right)=[M]$.

Notations: (i) For integers $n \geq m \geq 0$ we set $L_{n, m}:=\mathbb{Q}\left(\mu_{p^{n}}, \sqrt[p^{m}]{\Delta}\right)$, and denote by $\operatorname{reg}_{n, m}$ the regular representation of $\operatorname{Gal}\left(L_{n, m} / \mathbb{Q}\right)$. One can then define

$$
s_{n, m}(E):=\operatorname{corank}_{\mathbb{Z}_{p}} \operatorname{Sel}_{p^{\infty}}\left(E / L_{n, m}\right)+p^{\min (\delta, m)} \times \frac{1+a_{p}(E)}{2}
$$

which is the $p^{\infty}$-Selmer corank for $E$ over $L_{n, m}$, increased by the trivial zero contribution (indeed we shall show later in Section 3 that the quantity $p^{\min (\delta, m)}$ coincides with the number of places $\nu$ of $L_{n, m}$ lying over $p$ ).

(ii) Under the assumption that $M$ belongs to the category $\mathfrak{M}_{\mathcal{H}}\left(G_{\infty}\right)$ the Pontryagin dual of $\operatorname{Sel}_{p \infty}\left(E / L_{\infty, m}\right)$ is a cotorsion $\mathbb{Z}_{p}\left[\left[U^{(n)}\right]\right]$-module, in which case one may define the algebraic $\lambda$-invariant $\lambda_{L_{n, m}}^{\text {alg }}(E)$ to be the $\lambda_{\mathbb{Z}_{p}\left[\left[U^{(n)}\right]\right]}$-invariant of $\operatorname{Sel}_{p \infty}\left(E / L_{\infty, m}\right)^{\wedge}$.

(iii) Lastly for an Artin representation $\rho$ factoring through $G_{\infty}$, we will henceforth write $\Phi_{\rho}^{\prime}: \mathrm{K}_{1}\left(\mathbb{Z}_{p}\left[\left[G_{\infty}\right]\right]_{\mathcal{S}^{*}}\right) \rightarrow \operatorname{Quot}\left(\mathbb{Z}_{p}\left[\left[U^{(1)}\right]\right]\right)^{\times}$for the 'evaluation-at- $\rho$ map' in [4, Eqn (22)].

Theorem 7. (a) There are inequalities $s_{n, m}(E) \leq \operatorname{order}_{T=0}\left(\Phi_{\operatorname{reg}_{n, m}}^{\prime}\left(\xi_{M}\right)\right) \leq \lambda_{L_{n, m}}^{\text {alg }}(E)$.

(b) If $\operatorname{Sel}_{p \infty}\left(E / L_{\infty, m}\right)^{\wedge}$ has a semi-simple $\Lambda$-structure, one has a higher derivative formula

$$
\begin{aligned}
& \frac{1}{s_{n, m}(E) !} \cdot \frac{\mathrm{d}^{s_{n, m}(E)} \Phi_{\mathrm{reg}_{n, m}}^{\prime}\left(\xi_{M}\right)}{\mathrm{d} T^{s_{n, m}(E)}} \equiv \ell_{p}(E) \times \# \mathbf{I I}\left(E / L_{n, m}\right)_{p^{\infty}} \times \operatorname{det}\left(\langle-,-\rangle_{L_{n, m}}\right) \\
& \times\left(p^{n p^{\min (\delta, m)}}\right)^{\frac{1+a_{p}(E)}{2}} \times \prod_{\nu \mid \Delta} L_{\nu}(E, 1) \times \prod_{\nu \nmid \infty} \operatorname{Tam}_{\nu}\left(E / L_{n, m}\right) \times \# E\left(L_{n, m}\right)_{\text {tors }}^{-2}
\end{aligned}
$$

up to a p-adic unit, where the $\ell$-invariant $\ell_{p}(E):= \begin{cases}\prod_{\nu \mid p} \frac{\log _{p}\left(q_{E, \nu}\right)}{\operatorname{ord}_{\nu}\left(q_{E, \nu}\right)} & \text { if } a_{p}(E)=+1 \\ 1 & \text { if } a_{p}(E)=-1 .\end{cases}$ 
Applying the main theorem of [1], the $\ell$-invariant term is non-zero as the Tate period $q_{E, \nu}$ associated to a rigid analytic parametrization for $E$ at each $\nu \mid p$ of $L_{n, m}$ is transcendental. In fact the order of vanishing in $7(\mathrm{~b})$ above will become equal to $s_{n, m}(E)$, if and only if (i) the $p$-primary part of the Tate-Shafarevich group $\mathbf{I I I}\left(E / L_{n, m}\right)$ for $E$ over $L_{n, m}$ is finite, and (ii) the $p$-adic height pairing $\langle-,-\rangle_{L_{n, m}}: E\left(L_{n, m}\right) \times E\left(L_{n, m}\right) \rightarrow \mathbb{Q}_{p}$ constructed by Schneider and Jones $[19,20,14]$ is a non-degenerate form.

Conjecture 8. $\quad s_{n, m}(E)=\operatorname{order}_{T=0}\left(\Phi_{\operatorname{reg}_{n, m}}^{\prime}\left(\xi_{M}\right)\right)$.

We have at least one small fragment of evidence in support of this prediction.

Theorem 9. If $\operatorname{Sel}_{p}\left(E / L_{n, m}\right)$ is finite, then Conjecture 8 holds true.

In Section 3.3 we shall formulate a 'Main Conjecture' linking the characteristic element $\xi_{M}$ with the abelian $p$-adic $L$-functions constructed in Theorem 1 . In the non-split case the conjecture is straightforward to state. However in the split case $a_{p}(E)=+1$ not only do we encounter extra powers of $T$ caused by the exceptional zero condition, but also dual factors $\mathcal{D}_{p}(\rho, T)$ occurring at those same Artin representations $\rho$ which produce exceptional zeros in $\mathbf{L}_{p}(E, \rho)$ (note the $\mathcal{D}_{p}$-factors themselves are non-vanishing at $T=0$ ).

A Numerical Example. Consider the elliptic curve E15a1 given by the Weierstrass equation $E: y^{2}+x y+y=x^{3}+x^{2}-10 x-10$, with non-split multiplicative reduction at $p=3$. Choosing $\Delta=2$ then $\delta=\operatorname{ord}_{3}\left(2^{2}-1\right)-1=0$, while $K_{1}=\mathbb{Q}\left(\mu_{3}\right)$ and $L_{1}=\mathbb{Q}\left(\mu_{3}, \sqrt[3]{2}\right)$. Evaluating $L\left(E, \rho_{1}, s\right)$ at $s=1$ yields

$$
L\left(E, \rho_{1}, 1\right) \approx 1.72104398080992
$$

and then using in-built MAGMA functions, one calculates that

$$
L^{*}\left(E, \rho_{1}\right):=\left|\frac{L\left(E, \rho_{1}, 1\right) \sqrt{\operatorname{disc}_{K_{1}}}}{\left(2 \Omega_{E}^{+} \Omega_{E}^{-}\right)^{(3-1) / 2}}\right| \approx 4.00000000000001
$$

Note that we make the approximation $L^{*}\left(E, \rho_{1}\right) \approx 4$ as we do not expect $L^{*}\left(E, \rho_{1}\right)$ to be divisible by large primes (for the small $\Delta$ 's occurring in our computations).

As $f\left(\rho_{1}, 3\right)=3$ we know $a_{3}(E)^{f\left(\rho_{1}, 3\right)}=-1$, and the local $L$-factor for $L\left(E, \rho_{1}, s\right)$ at the prime 2 is given by $P_{2}\left(E, \rho_{1}, 2^{-1}\right)=1$. Using the Dokchitsers' technique [11, $\left.\S 6.10\right]$ for the local epsilon factors, one finds $\epsilon_{F_{1}}\left(\rho_{1}\right)_{3} \approx-1.04520385168448 E-14+5.19615242270663 i$ in which case $\epsilon_{F_{1}}\left(\rho_{1}\right)_{3} / \sqrt{\operatorname{disc}_{\mathbb{Q}(\sqrt[3]{2})}} \approx \frac{i}{2}$. Compiling this information, one obtains

$$
\mathbf{1}\left(\mathbf{L}_{p}\left(E, \rho_{1}\right)\right)=L^{*}\left(E, \rho_{1}\right) \times \frac{2^{(3-1) / 2}}{\sqrt{\operatorname{disc}_{\mathbb{Q}(\sqrt[3]{2})}}} P_{2}\left(E, \rho_{1}, 2^{-1}\right) \frac{\epsilon_{F_{1}}\left(\rho_{1}\right)_{3}}{a_{3}(E)^{f\left(\rho_{1}, 3\right)}} \approx 4+O\left(3^{8}\right) .
$$

Let us now consider $\sigma=\operatorname{Ind}_{K_{1}}^{\mathbb{Q}}(\mathbf{1}) \cong \mathbf{1} \oplus \omega$. Evaluating $L(E, \sigma, s)$ at $s=1$, we compute that $L(E, \sigma, 1) \approx 0.322695746401859$. Again exploiting the in-built MAGMA functions:

$$
L^{*}(E, \sigma):=\left|\frac{L(E, \sigma, 1) \sqrt{\operatorname{disc}_{F_{1}}}}{\left(2 \Omega_{E}^{+} \Omega_{E}^{-}\right)^{\frac{(3-1)}{2}}}\right| \approx 0.125000000000000=\frac{1}{8} .
$$


Furthermore $\epsilon_{F_{1}}(\sigma)_{3} \approx-2.66453525910038 E-15+1.73205080756888 i$ and $\frac{\epsilon_{F_{1}}(\sigma)_{3}}{\sqrt{\operatorname{disc}_{K_{1}}}} \approx-1$, whilst $P_{2}\left(E, \sigma, 2^{-1}\right)=2$. Lastly since $f(\sigma, 3)=1$, one therefore has $a_{3}(E)^{f(\sigma, 3)}=-1$. Putting all of this together, we deduce that

$$
\mathbf{1}\left(\mathbf{L}_{3}(E, \sigma)\right)=L^{*}(E, \sigma) \times P_{2}\left(E, \sigma, 2^{-1}\right) \frac{2^{(3-1) / 2}}{\sqrt{\operatorname{disc}_{K_{1}}}} \frac{2 \epsilon_{F_{1}}(\sigma)_{3}}{a_{3}(E)^{f(\sigma, 3)}} \approx 1+O\left(3^{8}\right) .
$$

As a consequence $\mathbf{1}\left(\mathbf{L}_{p}\left(E, \rho_{1}\right)\right)=4+O\left(3^{8}\right) \equiv 1+O\left(3^{8}\right)=\mathbf{1}\left(\mathbf{L}_{3}(E, \sigma)\right)$ modulo 3, which is equivalent to the first layer congruence $a_{1} \equiv N_{0,1}\left(a_{0}\right)$ modulo 3 at the trivial character.

\section{The Analytic Side}

We begin by recalling some background facts from the theory of Hilbert modular forms. Let $F$ be a totally real field such that $F / \mathbb{Q}$ is abelian. Following the notation from [18], set $h=\left|C l^{\dagger}(F)\right|$ to be the narrow class number of $F$, and choose ideles $t_{1}, \ldots, t_{h}$ such that $\tilde{t}_{\lambda} \triangleleft \mathcal{O}_{F}$ (the ideals generated by the $t_{\lambda}$ ) are all prime to $p$, and form a complete set of representatives for $C l^{\dagger}(F)$. We also denote the different of $F / \mathbb{Q}$ by $\mathfrak{d}_{F}$.

Hilbert automorphic forms over $F$ are holomorphic functions $\mathbf{f}: \mathrm{GL}_{2}\left(\mathbb{A}_{F}\right) \longrightarrow \mathbb{C}$ satisfying certain automorphy properties (see [18] or [23] for details). They also correspond to $h$-tuples $\left(f_{1}, \ldots f_{h}\right)$ of Hilbert modular forms on $\mathcal{H}^{d}$ where $d=[F: \mathbb{Q}]$. If $\mathbf{f} \in \mathcal{M}_{k}(\mathfrak{c}, \psi)$ (the set of Hilbert automorphic forms of parallel weight $k$, level $\mathfrak{c}$ and character $\psi$ ) then

$$
\left.f_{\lambda}\right|_{k} \gamma=\psi(\gamma) f_{\lambda}
$$

for $\lambda=1, \ldots, h$ and all $\gamma \in \Gamma_{\lambda}(\mathfrak{c})$, with

$$
\Gamma_{\lambda}(\mathfrak{c})=\left\{\left(\begin{array}{ll}
a & b \\
c & d
\end{array}\right): b \in \tilde{t}_{\lambda}^{-1} \mathfrak{d}_{F}^{-1}, c \in \tilde{t}_{\lambda} \mathfrak{c} \mathfrak{d}_{F}, a, d \in \mathcal{O}_{F}, a d-b c \in \mathcal{O}_{F}^{\times}\right\} .
$$

We define

$$
e_{F}(\xi z)=\exp \left(2 \pi i \sum_{1 \leq a \leq d} \xi^{\tau_{a}} z_{a}\right)
$$

where $z=\left(z_{1}, \ldots, z_{d}\right) \in \mathcal{H}^{d}, \xi \in F$ and $\tau_{1}, \ldots, \tau_{d}$ are the distinct embeddings $F \hookrightarrow \mathbb{R}$. Then, each component $f_{\lambda}$ has a Fourier expansion of the form

$$
f_{\lambda}(z)=\sum_{\xi} a_{\lambda}(\xi) e_{F}(\xi z)
$$

where the sum is taken over all totally positive $\xi \in \tilde{t}_{\lambda}$ and $\xi=0$. If $\mathbf{f}$ is a cusp form, then $a_{\lambda}(0)=0$ for all $\lambda$. The set of cusp forms of parallel weight $k$, level $\mathfrak{c}$ and character $\psi$ is written $\mathcal{S}_{k}(\mathfrak{c}, \psi)$. The form $\mathbf{f}$ itself also has Fourier coefficients $C(\mathfrak{m}, \mathbf{f})$ which satisfy

$$
C(\mathfrak{m}, \mathbf{f})= \begin{cases}a_{\lambda}(\xi) N_{F / \mathbb{Q}}\left(\tilde{t}_{\lambda}\right)^{-k / 2} & \text { if the ideal } \mathfrak{m}=\xi \tilde{t}_{\lambda}^{-1} \text { is integral } \\ 0 & \text { if } \mathfrak{m} \text { is not integral. }\end{cases}
$$

We will employ certain linear operators on the space of Hilbert automorphic forms. 
Definition 10. Let $\mathfrak{q}$ be an integral ideal of $\mathcal{O}_{F}$, and $q$ an idele for which $\tilde{q}=\mathfrak{q}$. We define the operators $\mathfrak{q}$ and $U(\mathfrak{q})$ on $\mathbf{f} \in \mathcal{M}_{k}(\mathfrak{c}, \psi)$ :

$$
\begin{aligned}
(\mathbf{f} \mid \mathfrak{q})(x) & =N_{F / \mathbb{Q}}(\mathfrak{q})^{-k / 2} \mathbf{f}\left(x\left(\begin{array}{cc}
q & 0 \\
0 & 1
\end{array}\right)\right) \\
(\mathbf{f} \mid U(\mathfrak{q}))(x) & =N_{F / \mathbb{Q}}(\mathfrak{q})^{k / 2-1} \sum_{v \in \mathcal{O}_{F} / \mathfrak{q}} \mathbf{f}\left(x\left(\begin{array}{cc}
1 & v \\
0 & q
\end{array}\right)\right) .
\end{aligned}
$$

These maps may also be described by their effect on the Fourier coefficients of $\mathbf{f}$, i.e.

$$
C(\mathfrak{m}, \mathbf{f} \mid \mathfrak{q})=C\left(\mathfrak{m} \mathfrak{q}^{-1}, \mathbf{f}\right) \quad \text { and } \quad C(\mathfrak{m}, \mathbf{f} \mid U(\mathfrak{q}))=C(\mathfrak{m} \mathfrak{q}, \mathbf{f})
$$

We also use the operator $J_{\mathfrak{c}}$, which is defined by

$$
\left(\mathbf{f} \mid J_{\mathfrak{c}}\right)(x)=\psi\left(\operatorname{det}(x)^{-1}\right) \mathbf{f}\left(x\left(\begin{array}{cc}
0 & 1 \\
c_{0} & 0
\end{array}\right)\right)
$$

where $c_{0}$ is an idele with $\tilde{c}_{0}=\mathfrak{c} \mathfrak{d}_{F}^{2}$. Then $\mathbf{f} \mid J_{\mathfrak{c}} \in \mathcal{M}_{k}\left(\mathfrak{c}, \psi^{-1}\right)$, and $\mathbf{f} \mid J_{\mathfrak{c}}^{2}=\mathbf{f}$ if $k$ is even. This mapping has the additional property

$$
\mathbf{f}\left|J_{\mathfrak{m} \mathfrak{c}}=N_{F / \mathbb{Q}}(\mathfrak{m})^{k / 2}\left(\mathbf{f} \mid J_{\mathfrak{c}}\right)\right| \mathfrak{m} .
$$

Further, when $\mathbf{f}$ is a primitive form in $\mathcal{M}_{k}(\mathfrak{c}, \psi)$, we have $\mathbf{f} \mid J_{\mathfrak{c}}=\Lambda(\mathbf{f}) \mathbf{f}^{\iota}$ where $\Lambda(\mathbf{f})$ is a root of unity, and $\mathbf{f}^{\iota}$ is the form with Fourier coefficients $C\left(\mathfrak{m}, \mathbf{f}^{\iota}\right)=\overline{C(\mathfrak{m}, \mathbf{f})}$.

Remarks: (i) If $f_{E} \in \mathcal{S}_{2}^{\text {new }}\left(\Gamma_{0}\left(N_{E}\right)\right)$ is the newform associated to $E_{/ \mathbb{Q}}$, we write $\mathbf{f}_{E}$ for the base change of $f_{E}$ to the totally real field $F$, with trivial character and conductor $\mathfrak{c}\left(\mathbf{f}_{E}\right)$. Assuming $F / \mathbb{Q}$ is abelian, this is the Hilbert modular form whose $L$-series satisfies

$$
L\left(s, \mathbf{f}_{E}\right)=\prod_{\psi \in \hat{G}} L(E, \psi, s) \quad \text { where } G=\operatorname{Gal}(F / \mathbb{Q}) .
$$

(ii) For each character $\chi: \operatorname{Gal}\left(\mathbb{Q}_{F T} / F_{n}\right) \rightarrow \mathbb{C}^{\times}$, we will write $\chi^{\dagger}: \mathcal{I}_{F_{n}} \rightarrow \mathbb{C}^{\times}$for the character of ideals associated to $\chi$ via composition with the reciprocity map; specifically $\chi^{\dagger}$ is normalised by $\chi^{\dagger}(\mathfrak{q})=\chi\left(\right.$ Frob $\left._{\mathfrak{q}}\right)$ for all primes $\mathfrak{q}$ of $F_{n}$, where Frob $\mathfrak{q}_{\mathfrak{q}}$ denotes an arithmetic Frobenius element at $\mathfrak{q}$.

Let $K / F$ be a totally imaginary quadratic extension. The following is due to Serre [21]:

Theorem 11. If $\rho$ is an Artin representation over $F$ which is induced from the Hecke character $\chi_{\rho}$ over $K$, then there exists a Hilbert automorphic form $\mathbf{g}_{\rho}$ over $F$ such that $\mathbf{g}_{\rho} \in \mathcal{S}_{1}\left(\mathfrak{c}\left(\mathbf{g}_{\rho}\right),(\operatorname{det} \rho)^{\dagger}\right)$ and

$$
L\left(s, \mathbf{g}_{\rho}\right)=L(s, \rho) .
$$

Further, $\mathbf{g}_{\rho}$ is primitive if and only if $\chi_{\rho}$ is a primitive character.

It is easily checked that the Fourier coefficients of $\mathbf{g}_{\rho}$ are

$$
C\left(\mathfrak{m}, \mathbf{g}_{\rho}\right)=\sum_{\substack{\mathfrak{a} \leq \mathcal{O}_{K}, \mathfrak{a} \overline{\mathfrak{a}}=\mathfrak{m}}} \chi_{\rho}^{\dagger}(\mathfrak{a}) .
$$


Also in the case $F=F_{k}, K=K_{k}$ and $\rho=\rho_{k}$, we assumed $\operatorname{gcd}\left(\Delta, N_{E}\right)=1$ which implies that $\mathfrak{p}^{-1} \mathfrak{c}\left(\mathbf{f}_{E}\right)$ and $\mathfrak{c}\left(\mathbf{g}_{\rho_{k}}\right)$ are coprime ideals of $\mathcal{O}_{F_{k}}$. The character $(\operatorname{det} \rho)^{\dagger}$ satisfies

$$
(\operatorname{det} \rho)^{\dagger}(\mathfrak{a})=\theta_{K / F}(\mathfrak{a}) \chi_{\rho}^{\dagger}\left(\mathfrak{a} \mathcal{O}_{K}\right)
$$

where $\theta_{K / F}$ is the quadratic character of $K / F$, given on prime ideals of $\mathcal{O}_{F}$ by

$$
\theta_{K / F}(\mathfrak{q})= \begin{cases}1 & \text { if } \mathfrak{q} \text { splits in } K / F \\ -1 & \text { if } \mathfrak{q} \text { is inert in } K / F \\ 0 & \text { if } \mathfrak{q} \text { ramifies in } K / F\end{cases}
$$

We use a non-standard normalisation $[18$, Chap $4, \S 1.4]$ of the Petersson inner product,

$$
\langle\mathbf{F}, \mathbf{G}\rangle_{\mathfrak{c}}:=\sum_{\lambda=1}^{h} \int_{\Gamma_{\lambda}(\mathfrak{c}) \backslash \mathcal{H}^{d}} \overline{\mathbf{F}_{\lambda}(z)} \mathbf{G}_{\lambda}(z) N(y)^{k} \mathrm{~d} \nu(z)
$$

where $\mathbf{F} \in \mathcal{S}_{k}(\mathfrak{c}, \psi), \mathbf{G} \in \mathcal{M}_{k}(\mathfrak{c}, \psi), d=[F: \mathbb{Q}]$ and $\mathrm{d} \nu(z)=\prod_{1 \leq j \leq d} y_{j}^{-2} \mathrm{~d} x_{j} \mathrm{~d} y_{j}$.

\subsection{Constructing the distribution}

Let $\rho$ be a 2-dimensional Artin representation over $F$ factoring through a subgroup of $G_{\infty}$. For example, $\rho$ might correspond to the representation induced from a Hecke character over a CM extension of $F$ (e.g. character $\chi_{\rho_{k}}$ in the Introduction) with theta-series $\mathbf{g}_{\rho}$. Consider the finite set of primes $S=\{v: v$ is a prime of $F, v \mid \Delta\}$; we shall study the value at $s=1$ of the normalised Rankin-Selberg product

$$
\Psi\left(s, \mathbf{f}_{E}, \mathbf{g}_{\rho}\right)=\left(\frac{\Gamma(s)}{(2 \pi)^{s}}\right)^{2[F: \mathbb{Q}]} L_{\mathfrak{c}}\left(2 s-1,(\operatorname{det} \rho)^{\dagger}\right) L\left(s, \mathbf{f}_{E}, \mathbf{g}_{\rho}\right)
$$

where $\mathfrak{c}=\mathfrak{c}\left(\mathbf{f}_{E}\right) \mathfrak{c}\left(\mathbf{g}_{\rho}\right)$, and $L\left(s, \mathbf{f}_{E}, \mathbf{g}_{\rho}\right)=\sum_{\mathfrak{a}} C\left(\mathfrak{a}, \mathbf{f}_{E}\right) C\left(\mathfrak{a}, \mathbf{g}_{\rho}\right) N_{F / \mathbb{Q}}(\mathfrak{a})^{-s}$.

We need a few preparatory lemmas, starting with a result on the epsilon factor $\epsilon_{F}(s, \rho)$. The Artin $L$-function $L(s, \rho)$ obeys the functional equation

$$
\Gamma_{\infty}(s) L(s, \rho)=\epsilon_{F}(s, \rho) \Gamma_{\infty}(1-s) L\left(1-s, \rho^{\vee}\right)
$$

where $\rho^{\vee}$ indicates the contragredient representation, and $\Gamma_{\infty}(s):=\left((2 \pi)^{-s} \Gamma(s)\right)^{[F: \mathbb{Q}]}$. The global $\epsilon$-factor at zero may be decomposed into an infinite product

$$
\epsilon_{F}(0, \rho)=\prod_{\text {all places } v} \epsilon_{F_{v}}\left(\rho_{v}, \psi_{\nu}, d x_{\nu}\right)
$$

where each local factor depends on the normalisation of additive characters $\psi_{\nu}$, and Haar measures $d x_{\nu}$ (however the product does not).

Lemma 12. Setting $\epsilon_{F}(\rho)=\epsilon_{F}(0, \rho)$, we have

$$
\Lambda\left(\mathbf{g}_{\rho}\right)=i^{-[F: \mathbb{Q}]} N_{F / \mathbb{Q}}\left(\mathfrak{c}\left(\mathbf{g}_{\rho}\right) \mathfrak{d}_{F}^{2}\right)^{-1 / 2} \epsilon_{F}(\rho) .
$$

Proof. This is [7, Lemma 2.2] though there is a typographical error: $\mathfrak{c}$ should be $\mathfrak{c}\left(\mathbf{g}_{\rho}\right)$. 
We will use the following integral representation, a special case of [23, Equation (4.32)].

Proposition 13. Let $\mathbf{F}, \mathbf{G}$ be HMFs such that $\mathbf{F}$ is a cusp form and $\mathbf{G}$ has character $\omega$. If the $\mathcal{O}_{F}$-ideal $\mathfrak{c} \subset \mathfrak{c}(\mathbf{F}) \mathfrak{c}(\mathbf{G})$ then

$$
\Psi\left(1, \mathbf{F}, \mathbf{G}^{\iota}\right)=D_{F}^{1 / 2} \pi^{-[F: \mathbb{Q}]}\left\langle\mathbf{F}^{\iota}, V(0)\right\rangle_{\mathfrak{c}}
$$

where $D_{F}$ denotes the field discriminant of $F / \mathbb{Q}$, and $V(0):=\mathbf{G}^{\iota} \cdot \mathcal{K}_{1}^{0}\left(0 ; \mathfrak{c}, \mathcal{O}_{F} ; \omega^{-1}\right)$ with $\mathcal{K}_{1}^{0}$ the Eisenstein series given in [18, Chapter 4, (4.1)] whose $\lambda$-components are

$$
\mathcal{K}_{1}^{0}\left(0 ; \mathfrak{c}, \mathcal{O}_{F} ; \omega\right)_{\lambda}(z)=N_{F / \mathbb{Q}}\left(\tilde{t}_{\lambda}\right)^{1 / 2} \sum_{c, d} \operatorname{sign}\left(N_{F / \mathbb{Q}}(d)\right) \omega^{*}\left(d \mathcal{O}_{F}\right) N_{F / \mathbb{Q}}(c z+d)^{-1} .
$$

Note that the sum is taken over the set of equivalence classes

$$
(c, d) \in \frac{\tilde{t}_{\lambda} \mathfrak{d}_{F} \mathfrak{c} \times \mathcal{O}_{F}}{\sim}
$$

where the relation $\sim$ is defined by $(c, d) \sim(u c, u d)$ for all $u \in \mathcal{O}_{F}^{\times}$.

It is useful to convert $\mathcal{K}_{1}^{0}$ to an Eisenstein series which has a user-friendly Fourier expansion; we can do this via the involution $J_{\mathfrak{c}}$. If $\omega=(\operatorname{det} \rho)^{\dagger}$ then using $[18$, Chapter 4 , (4.6)],

$$
\mathcal{K}_{1}^{0}\left(0 ; \mathfrak{c}, \mathcal{O}_{F} ;(\operatorname{det} \rho)^{\dagger-1}\right) \mid J_{\mathfrak{c}}=\frac{(4 \pi i)^{[F: \mathbb{Q}]}}{D_{F}^{1 / 2} N_{F / \mathbb{Q}}\left(\mathfrak{c}\left(\mathbf{g}_{\rho}\right) \mathfrak{d}_{F}^{2}\right)^{1 / 2}} E_{1}\left(0, \mathfrak{c},(\operatorname{det} \rho)^{\dagger-1}\right) .
$$

Here $E_{1}$ is the Eisenstein series in [18, Chapter 4, (4.13)], with $\lambda$-components

$$
E_{1}(0, \mathfrak{c}, \omega)_{\lambda}(z)=\frac{N_{F / \mathbb{Q}}\left(\tilde{t}_{\lambda}\right)^{-1 / 2} D_{F}^{1 / 2}}{(-4 \pi i)^{[F: \mathbb{Q}]}} \sum_{c, d} \operatorname{sign}\left(N_{F / \mathbb{Q}}(c)\right) \omega^{*}\left(c \mathcal{O}_{F}\right) N_{F / \mathbb{Q}}(c z+d)^{-1}
$$

so that $\omega$ is viewed an ideal character modulo $\mathfrak{c}$, and the sum ranges over

$$
(c, d) \in \frac{\mathcal{O}_{F} \times \tilde{t}_{\lambda}^{-1} \mathfrak{d}_{F}^{-1}}{\sim} .
$$

The Fourier expansion of each $\lambda$-component is computed in [18, Chapter 4, Prop 4.2], namely

$$
E_{1}\left(0, \mathfrak{c},(\operatorname{det} \rho)^{\dagger-1}\right)_{\lambda}(z)=N_{F / \mathbb{Q}}\left(\tilde{t}_{\lambda}\right)^{-1 / 2} \sum_{0 \ll \xi \in \tilde{t}_{\lambda}} a_{\lambda}(\xi) e_{F}(\xi z)
$$

with each coefficient

$$
a_{\lambda}(\xi)=\sum_{\substack{\xi=\tilde{\tilde{o}} \tilde{c}, c \in \tilde{F}_{F}, b \in \hat{t}_{\lambda}}}(\operatorname{det} \rho)^{\dagger-1}(\tilde{c})
$$

For a finite place $v$ of $F$ that is coprime to $\mathfrak{c}\left(\mathbf{f}_{E}\right)$, we label roots $\alpha(v), \alpha^{\prime}(v)$ of the polynomial

$$
X^{2}-C\left(v, \mathbf{f}_{E}\right) X+N_{F / \mathbb{Q}}(v)=(X-\alpha(v))\left(X-\alpha^{\prime}(v)\right) ;
$$

we also define $\alpha(\mathfrak{p})=a_{p}(E)$ and $\alpha^{\prime}(\mathfrak{p})=0$. From these definitions, we extend $\alpha(\mathfrak{m}), \alpha^{\prime}(\mathfrak{m})$ multiplicatively to all ideals $\mathfrak{m}$ of $\mathcal{O}_{F}$. 
Definition 14. Set $\mathfrak{l}_{0}:=\prod_{\mathfrak{q} \mid \Delta} \mathfrak{q}$. Then the $\mathfrak{l}_{0}$-stabilisation of $\mathbf{f}_{E}$ is defined to be

$$
\mathbf{f}_{0}:=\sum_{\mathfrak{a} \mid \mathfrak{l}_{0}} M(\mathfrak{a}) \alpha^{\prime}(\mathfrak{a}) \cdot \mathbf{f}_{E} \mid \mathfrak{a}
$$

where $M$ is the Möbius function on ideals.

Following [18, Chapter 4, (3.14)], define

$$
\mathbf{g}_{\rho, \mathfrak{p r}}:=\sum_{\mathfrak{n} \mid \mathfrak{p r} \mathfrak{r}_{0}} M(\mathfrak{n}) \cdot \mathbf{g}_{\rho} \mid U(\mathfrak{n}) \circ \mathfrak{n} .
$$

In particular, $\mathbf{g}_{\rho, \mathfrak{p l}} \in \mathcal{M}_{1}\left(\mathfrak{c}\left(\mathbf{g}_{\rho}\right) \mathfrak{p}^{2} \mathfrak{l}_{0}^{2},(\operatorname{det} \rho)^{\dagger}\right)$ where $\mathfrak{c}\left(\mathbf{g}_{\rho}\right)$ was the conductor of $\mathbf{g}_{\rho}$.

Set $\mathfrak{c}_{0}=\mathfrak{l}_{0} \mathfrak{c}\left(\mathbf{f}_{E}\right)$; we shall choose $\mathcal{O}_{F}$-ideals $\mathfrak{m}^{\prime}$ and $\mathfrak{l}^{\prime}$ such that $\mathfrak{m}^{\prime}$ is a power of $\mathfrak{p}$, $\operatorname{supp}\left(\mathfrak{l}^{\prime}\right)=\operatorname{supp}\left(\mathfrak{l}_{0}\right)$ and $\mathfrak{c}\left(\mathbf{g}_{\rho}\right) \mathfrak{p}^{2} \mathfrak{l}_{0}^{2} \mid \mathfrak{m}^{\prime} \mathfrak{l}^{\prime}$. Clearly $\mathbf{f}_{0} \in \mathcal{S}_{\mathbf{2}}\left(\mathfrak{c}_{0}\right) \subset \mathcal{S}_{\mathbf{2}}\left(\mathfrak{c}\left(\mathbf{f}_{E}\right) \mathfrak{m}^{\prime} \mathfrak{l}^{\prime}\right)$, in fact

$$
\mathbf{g}_{\rho, \mathfrak{p} \mathfrak{l}_{0}} \in \mathcal{M}_{\mathbf{1}}\left(\mathfrak{c}\left(\mathbf{g}_{\rho}\right) \mathfrak{p}^{2} \mathfrak{l}_{0}^{2},(\operatorname{det} \rho)^{\dagger}\right) \subset \mathcal{M}_{\mathbf{1}}\left(\mathfrak{c}\left(\mathbf{f}_{E}\right) \mathfrak{m}^{\prime} \mathfrak{l}^{\prime},(\operatorname{det} \rho)^{\dagger}\right) .
$$

Now the associated contragredient Euler factor is defined by

$$
\begin{aligned}
\operatorname{Eul}_{\mathfrak{p r _ { 0 }}}\left(\rho^{\vee}, s\right):= & \prod_{v \mid \mathfrak{p r}_{0}}\left(1-\alpha^{\prime}(v) \hat{\beta}(v) N(v)^{-s}\right)\left(1-\alpha^{\prime}(v) \hat{\beta}^{\prime}(v) N(v)^{-s}\right) \\
& \times\left(1-\alpha^{-1}(v) \beta(v) N(v)^{s-1}\right)\left(1-\alpha^{-1}(v) \beta^{\prime}(v) N(v)^{s-1}\right)
\end{aligned}
$$

where we have factorised the Hecke polynomial for $\mathbf{g}_{\rho}$ as

$$
X^{2}-C\left(v, \mathbf{g}_{\rho}\right) X+(\operatorname{det} \rho)^{\dagger}(v)=(X-\beta(v))\left(X-\beta^{\prime}(v)\right)
$$

and likewise the dual Hecke polynomial via

$$
X^{2}-\overline{C\left(v, \mathbf{g}_{\rho}\right)} X+(\operatorname{det} \rho)^{\dagger-1}(v)=(X-\hat{\beta}(v))\left(X-\hat{\beta}^{\prime}(v)\right) .
$$

Lemma 15. There is an identity of Rankin-Selberg L-functions

$$
\begin{aligned}
\Psi\left(s, \mathbf{f}_{0}, \mathbf{g}_{\rho, \mathfrak{p} \mathfrak{p}_{0}} \mid J_{\mathfrak{c}\left(\mathbf{f}_{E}\right) \mathfrak{m}^{\prime} \mathfrak{l}^{\prime}}\right)= & N_{F / \mathbb{Q}}\left(\frac{\mathfrak{c}\left(\mathbf{f}_{E}\right) \mathfrak{m}^{\prime} \mathfrak{l}^{\prime}}{\mathfrak{c}\left(\mathbf{g}_{\rho}\right)}\right)^{1 / 2-s} \Lambda\left(\mathbf{g}_{\rho}\right) \alpha\left(\frac{\mathfrak{m}^{\prime} \mathfrak{l}^{\prime}}{\mathfrak{c}\left(\mathbf{g}_{\rho}\right)}\right) C\left(\mathfrak{c}\left(\mathbf{f}_{E}\right), \mathbf{f}_{E}\right) \\
& \times \operatorname{Eul}_{\mathfrak{p r}_{0}}\left(\rho^{\vee}, s\right) \times \Psi\left(s, \mathbf{f}_{E}, \mathbf{g}_{\rho}^{\iota}\right) .
\end{aligned}
$$

Because we assumed that $E$ is semistable over $\mathbb{Q}$, the coefficient

$$
C\left(\mathfrak{c}\left(\mathbf{f}_{E}\right), \mathbf{f}_{E}\right)=(-1)^{\# \mathcal{T}_{F}^{n s}} \neq 0
$$

where $\mathcal{T}_{F}^{n s}$ denotes the set of finite places where $E$ has non-split multiplicative reduction.

Proof. Recall that $\mathbf{f}_{E}\left|J_{\mathfrak{m} \mathfrak{c}}=N_{F / \mathbb{Q}}(\mathfrak{m})^{k / 2}\left(\mathbf{f}_{E} \mid J_{\mathfrak{c}}\right)\right| \mathfrak{m}$. Since $\mathfrak{c}\left(\mathbf{g}_{\rho, \mathfrak{p} \mathfrak{l}_{0}}\right)$ divides $\mathfrak{c}\left(\mathbf{g}_{\rho}\right) \mathfrak{p}^{2} \mathfrak{l}_{0}^{2}$, it follows that

$$
\mathbf{g}_{\rho, \mathfrak{p} \mathfrak{l}_{0}}\left|J_{\mathfrak{c}\left(\mathbf{f}_{E}\right) \mathfrak{m}^{\prime} \mathfrak{l}^{\prime}}=N_{F / \mathbb{Q}}\left(\frac{\mathfrak{c}\left(\mathbf{f}_{E}\right) \mathfrak{m}^{\prime} \mathfrak{l}^{\prime}}{\mathfrak{c}\left(\mathbf{g}_{\rho}\right) \mathfrak{p}^{2} \mathfrak{l}_{0}^{2}}\right)^{1 / 2} \cdot\left(\mathbf{g}_{\rho, \mathfrak{p} \mathfrak{r}_{0}} \mid J_{\mathfrak{c}\left(\mathbf{g}_{\rho}\right) \mathfrak{p}^{2} \mathfrak{l}_{0}^{2}}\right)\right| \frac{\mathfrak{c}\left(\mathbf{f}_{E}\right) \mathfrak{m}^{\prime} \mathfrak{l}^{\prime}}{\mathfrak{c}\left(\mathbf{g}_{\rho}\right) \mathfrak{p}^{2} \mathfrak{l}_{0}^{2}} .
$$


To avoid cramping our equations, we will write $\mathbf{f}=\mathbf{f}_{E}$ and $\mathbf{h}=\mathbf{g}_{\rho, \mathfrak{p} \mathfrak{p r}_{0}} \mid J_{\mathfrak{c}\left(\mathbf{g}_{\rho}\right) \mathfrak{p}^{2} \mathfrak{l}_{0}^{2}}$ so that

$$
\begin{aligned}
\Psi\left(s, \mathbf{f}_{0}, \mathbf{g}_{\rho, \mathfrak{p} \mathfrak{p}_{0}} \mid J_{\mathfrak{c}(\mathbf{f}) \mathfrak{m}^{\prime} \mathfrak{l}^{\prime}}\right) & =N_{F / \mathbb{Q}}\left(\frac{\mathfrak{c}(\mathbf{f}) \mathfrak{m}^{\prime} \mathfrak{l}^{\prime}}{\mathfrak{c}\left(\mathbf{g}_{\rho}\right) \mathfrak{p}^{2} \mathfrak{l}_{0}^{2}}\right)^{1 / 2} \Psi\left(s, \mathbf{f}_{0}, \mathbf{h} \mid \frac{\mathfrak{c}(\mathbf{f}) \mathfrak{m}^{\prime} \mathfrak{l}^{\prime}}{\mathfrak{c}\left(\mathbf{g}_{\rho}\right) \mathfrak{p}^{2} \mathfrak{l}_{0}^{2}}\right) \\
& =N_{F / \mathbb{Q}}\left(\frac{\mathfrak{c}(\mathbf{f}) \mathfrak{m}^{\prime} \mathfrak{l}^{\prime}}{\mathfrak{c}\left(\mathbf{g}_{\rho}\right) \mathfrak{p}^{2} \mathfrak{l}_{0}^{2}}\right)^{1 / 2-s} \Psi\left(s, \mathbf{f}_{0} \mid U\left(\frac{\mathfrak{c}(\mathbf{f}) \mathfrak{m}^{\prime} \mathfrak{l}^{\prime}}{\mathfrak{c}\left(\mathbf{g}_{\rho}\right) \mathfrak{p}^{2} \mathfrak{l}_{0}^{2}}\right), \mathbf{h}\right) \\
& =N_{F / \mathbb{Q}}\left(\frac{\mathfrak{c}(\mathbf{f}) \mathfrak{m}^{\prime} \mathfrak{l}^{\prime}}{\mathfrak{c}\left(\mathbf{g}_{\rho}\right) \mathfrak{p}^{2} \mathfrak{l}_{0}^{2}}\right)^{1 / 2-s} \alpha\left(\frac{\mathfrak{m}^{\prime} \mathfrak{l}^{\prime}}{\mathfrak{c}\left(\mathbf{g}_{\rho}\right) \mathfrak{p}^{2} \mathfrak{l}_{0}^{2}}\right) C(\mathfrak{c}(\mathbf{f}), \mathbf{f}) \Psi\left(s, \mathbf{f}_{0}, \mathbf{h}\right) .
\end{aligned}
$$

Here we have exploited the fact that $L\left(s, \mathbf{f}_{0}, \mathbf{g}_{\rho}^{\iota} \mid \mathfrak{a}\right)=N_{F / \mathbb{Q}}(\mathfrak{a})^{-s} L\left(s, \mathbf{f}_{0} \mid U(\mathfrak{a}), \mathbf{g}_{\rho}^{\iota}\right)$ for any ideal $\mathfrak{a}$, and also the formula

$$
\mathbf{f}_{0} \mid U\left(\frac{\mathfrak{c}(\mathbf{f}) \mathfrak{m}^{\prime} \mathfrak{l}^{\prime}}{\mathfrak{c}\left(\mathbf{g}_{\rho}\right) \mathfrak{p}^{2} \mathfrak{l}_{0}^{2}}\right)=\alpha\left(\frac{\mathfrak{m}^{\prime} \mathfrak{l}^{\prime}}{\mathfrak{c}\left(\mathbf{g}_{\rho}\right) \mathfrak{p}^{2} \mathfrak{l}_{0}^{2}}\right) C(\mathfrak{c}(\mathbf{f}), \mathbf{f}) \mathbf{f}_{0}
$$

which follows by construction of the $\mathfrak{p l}_{0}$-stabilisation $\mathbf{f}_{0}$. Note $C(\mathfrak{c}(\mathbf{f}), \mathbf{f})=a_{p}(E) \times C(\mathfrak{n}, \mathbf{f})$ where $\mathfrak{n}$ is the (square-free) tame conductor of $E / F$, and moreover

$$
\Psi\left(s, \mathbf{f}_{0}, \mathbf{h}\right)=N_{F / \mathbb{Q}}\left(\mathfrak{p}^{2} \mathfrak{l}_{0}^{2}\right)^{1-2 s} \alpha\left(\mathfrak{p}^{2} \mathfrak{l}_{0}^{2}\right) \Lambda\left(\mathbf{g}_{\rho}\right) \operatorname{Eul}_{\mathfrak{p} \mathfrak{l}_{0}}\left(\rho^{\vee}, s\right) \Psi\left(s, \mathbf{f}, \mathbf{g}_{\rho}^{\iota}\right) .
$$

Combining the two equations together yields the required result.

We introduce the trace map $\operatorname{Tr}_{\mathfrak{c}_{0}}^{\mathfrak{c}\left(\mathbf{f}_{E}\right) \mathfrak{m}^{\prime} \mathfrak{l}^{\prime}}: \mathcal{M}_{2}\left(\mathfrak{c}\left(\mathbf{f}_{E}\right) \mathfrak{m}^{\prime} \mathfrak{l}^{\prime}\right) \rightarrow \mathcal{M}_{2}\left(\mathfrak{c}_{0}\right)$ by

$$
\left(\mathbf{H} \mid \operatorname{Tr}_{\mathfrak{c}_{0}}^{\mathfrak{c}\left(\mathbf{f}_{E}\right) \mathfrak{m}^{\prime} \mathfrak{l}^{\prime}}\right)(x)=\sum_{v \in T} \mathbf{H}\left(x\left(\begin{array}{cc}
1 & 0 \\
c v & 1
\end{array}\right)\right)
$$

where $c$ is an idele such that $\tilde{c}=\mathfrak{c}_{0}$, and $T$ is the coset representatives for $\mathfrak{c}_{0} \mathcal{O}_{F} / \mathfrak{c}\left(\mathbf{f}_{E}\right) \mathfrak{m}^{\prime} \mathfrak{l}^{\prime}$. This map has the property that for every $\mathbf{F} \in \mathcal{S}_{2}\left(\mathfrak{c}_{0}\right)$,

$$
\langle\mathbf{F}, \mathbf{H}\rangle_{\mathfrak{c}\left(\mathbf{f}_{E}\right) \mathfrak{m}^{\prime} \mathfrak{l}^{\prime}}=\left\langle\mathbf{F}, \mathbf{H} \mid \operatorname{Tr}_{\mathfrak{c}_{0}}^{\mathfrak{c}\left(\mathbf{f}_{E}\right) \mathfrak{m}^{\prime} \mathfrak{l}^{\prime}}\right\rangle_{\mathfrak{c}_{0}} .
$$

Furthermore, from [18, Chapter 4, (4.11)] we have the formula

$$
\mathbf{H}\left|\operatorname{Tr}_{\mathfrak{c}_{0}}^{\mathfrak{c}\left(\mathbf{f}_{E}\right) \mathfrak{m}^{\prime} \mathfrak{l}^{\prime}}=\mathbf{H}\right| J_{\mathfrak{c}\left(\mathbf{f}_{E}\right) \mathfrak{m}^{\prime} \mathfrak{l}^{\prime}}\left|U\left(\mathfrak{m}^{\prime} \mathfrak{l}^{\prime} \mathfrak{r}_{0}^{-1}\right)\right| J_{\mathfrak{c}_{0}} .
$$

The latter arises from the definition of these operators, and the matrix identity

$$
\left(\begin{array}{cc}
1 & 0 \\
c v & 1
\end{array}\right)=(c m)^{-1}\left(\begin{array}{cc}
0 & 1 \\
c m & 0
\end{array}\right)\left(\begin{array}{cc}
1 & v \\
0 & m
\end{array}\right)\left(\begin{array}{ll}
0 & 1 \\
c & 0
\end{array}\right)
$$

which holds for any $c, m$ and $v$. If $\mathbf{H}=\Phi \mid J_{\mathfrak{c}\left(\mathbf{f}_{E}\right) \mathfrak{m}^{\prime} \mathfrak{l}^{\prime}}$, applying Equations (3) and (4) yields

$$
\left\langle\mathbf{f}_{0}{ }^{\iota}, \mathbf{H}\right\rangle_{\mathfrak{c}\left(\mathbf{f}_{E}\right) \mathfrak{m}^{\prime} \mathfrak{l}^{\prime}}=\left\langle\mathbf{f}_{0}{ }^{\iota}, \mathbf{H} \mid \operatorname{Tr}_{\mathfrak{c}_{0}} \mathfrak{c}^{\left(\mathbf{f}_{E}\right) \mathfrak{m}^{\prime} \mathfrak{l}^{\prime}}\right\rangle_{\mathfrak{c}_{0}}=\left\langle\mathbf{f}_{\mathbf{0}}{ }^{\iota}, \Phi\left|U\left(\mathfrak{m}^{\prime} \mathfrak{l}^{\prime} \mathfrak{r}_{0}^{-1}\right)\right| J_{\mathfrak{c}_{0}}\right\rangle_{\mathfrak{c}_{0}}
$$

The following definition differs slightly from its counterpart in [7] in that the periods we quotient by are motivic rather than automorphic. 
Definition 16. We define a $\mathbb{C}$-linear functional $\mathcal{L}_{F}$ on the complex vector space

$$
\mathcal{M}_{2}\left(\mathfrak{c}\left(\mathbf{f}_{E}\right) \mathfrak{m}^{\prime} \mathfrak{l}^{\prime}\right) \mid U\left(\mathfrak{m}^{\prime} \mathfrak{l}^{\prime} \mathfrak{r}_{0}^{-1}\right) \quad \text { by the rule } \quad \mathcal{L}_{F}: \Theta \longmapsto \frac{\left\langle\mathbf{f}_{\mathbf{0}}{ }^{\iota}, \Theta \mid J_{\mathfrak{c}_{0}}\right\rangle_{\mathfrak{c}_{0}}}{\left(\Omega_{E}^{+} \Omega_{E}^{-}\right)^{[F: \mathbb{Q}]}}
$$

Let $n \geq k$ be integers with $n \geq 1$ and $k \geq 0$, and put $F_{n}=\mathbb{Q}\left(\mu_{p^{n}}\right)^{+}$and $F_{k}=\mathbb{Q}\left(\mu_{p^{k}}\right)^{+}$ as in the Introduction. We shall consider the Hilbert automorphic form $\mathbf{g}_{\rho_{k} / F_{n}}$, i.e. the base change of $\mathbf{g}_{\rho_{k}}$ to $F_{n}$ - in a slight abuse of notation, we have elected to write $\rho_{k} / F_{n}$ as shorthand for the Artin representation $\operatorname{Res}_{F_{n}}\left(\rho_{k}\right)=\operatorname{Ind}_{K_{n}}^{F_{n}}\left(\operatorname{Res}_{K_{n}}\left(\chi_{\rho_{k}}\right)\right)$.

Let us denote by $\mathcal{G}_{n}$ the topological group $\operatorname{Gal}\left(F_{n, S}^{\mathrm{ab}} / F_{n}\right)$, where $F_{n, S}^{\mathrm{ab}}$ is the maximal abelian extension of $F_{n}$ unramified outside $S \cup\{\mathfrak{p}\}=\left\{v: v \mid \mathfrak{p l}_{0}\right\}$ and the infinite places. Fix a multiplicative character $\psi: \mathcal{G}_{n} \rightarrow \mathbb{C}^{\times}$of conductor $\mathfrak{f}_{\psi}$; as in [7, Definition 2.10] we introduce the automorphic form over $F=F_{n}$ :

$$
\begin{aligned}
\Phi_{\psi}^{n, k} & =\Phi_{\psi}^{n, k}\left(\rho_{k} / F_{n} \otimes \psi, \mathfrak{c}\left(\mathbf{f}_{E}\right) \mathfrak{m}^{\prime} \mathfrak{l}^{\prime}\right) \\
& :=\left(\mathbf{g}_{\rho_{k} / F_{n} \otimes \psi, \mathfrak{p} \mathfrak{r}_{0}}\right) \cdot E_{1}\left(0, \mathfrak{c}\left(\mathbf{f}_{E}\right) \mathfrak{m}^{\prime} \mathfrak{l}^{\prime},\left(\operatorname{Res}_{F_{n}}\left(\operatorname{det} \rho_{k}\right)\right)^{-1} \otimes \psi^{-2}\right)
\end{aligned}
$$

where we assume $\mathfrak{m}^{\prime}$ and $\mathfrak{l}^{\prime}$ satisfy $\mathfrak{c}\left(\mathbf{g}_{\rho}\right)\left(\mathfrak{p} \mathfrak{l}_{0} \mathfrak{f}_{\psi}\right)^{2} \mid \mathfrak{m}^{\prime} \mathfrak{l}^{\prime}$.

It follows from Proposition 13 and Equation (5) above that

$$
\frac{(-4 i)^{\left[F_{n}: \mathbb{Q}\right]}}{N_{F_{n} / \mathbb{Q}}\left(\mathfrak{c}\left(\mathbf{f}_{E}\right) \mathfrak{m}^{\prime} \mathfrak{l}^{\prime} \mathfrak{d}_{F}^{2}\right)^{1 / 2}} \mathcal{L}_{F_{n}}\left(\Phi_{\psi}^{n, k} \mid U\left(\mathfrak{m}^{\prime} \mathfrak{l}^{\prime} \mathfrak{l}_{0}^{-1}\right)\right)=\frac{\Psi\left(1, \mathbf{f}_{0}, \mathbf{g}_{\rho_{k} / F_{n} \otimes \psi, \mathfrak{p} \mathfrak{l}_{0}} \mid J_{\mathfrak{c}\left(\mathbf{f}_{E}\right) \mathfrak{m}^{\prime} \mathfrak{l}^{\prime}}\right)}{\left(\Omega_{E}^{+} \Omega_{E}^{-}\right)^{\left[F_{n}: \mathbb{Q}\right]}}
$$

Combining this with Lemma 15, we obtain the following relationship between $\Phi_{\psi}^{n, k}$ and the Rankin-Selberg $L$-function.

Proposition 17. For all integers $n \geq k$,

$$
\begin{aligned}
\frac{(-4 i)^{\phi\left(p^{n}\right) / 2}}{\alpha\left(\mathfrak{m}^{\prime} \mathfrak{l}^{\prime}\right) C\left(\mathfrak{c}\left(\mathbf{f}_{E}\right), \mathbf{f}_{E}\right)} \mathcal{L}_{F_{n}}\left(\Phi_{\psi}^{n, k} \mid U\left(\mathfrak{m}^{\prime} \mathfrak{l}^{\prime} \mathfrak{l}_{0}^{-1}\right)\right)=\frac{N_{F_{n} / \mathbb{Q}}\left(\mathfrak{c}\left(\mathbf{g}_{\rho_{k} / F_{n} \otimes \psi}\right) \mathfrak{d}_{F_{n}}^{2}\right)^{1 / 2}}{\alpha\left(\mathfrak{c}\left(\mathbf{g}_{\rho_{k} / F_{n} \otimes \psi}\right)\right)} \\
\times \Lambda\left(\mathbf{g}_{\rho_{k} / F_{n} \otimes \psi}\right) \times \operatorname{Eul}_{\mathfrak{p r} 1_{0}}\left(\rho_{k} / F_{n} \otimes \psi^{-1}, 1\right) \times \frac{\Psi\left(1, \mathbf{f}_{E}, \mathbf{g}_{\rho_{k} / F_{n} \otimes \psi}^{\iota}\right)}{\left(\Omega_{E}^{+} \Omega_{E}^{-}\right)^{\left[F_{n}: \mathbb{Q}\right]}}
\end{aligned}
$$

Furthermore, the Fourier coefficients of each $\lambda$-component of $\Phi_{\psi}^{n, k}$ are given by

$$
\begin{aligned}
\phi_{\psi, \lambda}^{n, k}(\xi) & =\sum_{\xi=\xi_{1}+\xi_{2}} \sum_{\substack{\mathfrak{a} \triangleleft \mathcal{O}_{K_{n}}, \mathfrak{a} \bar{a}=\xi_{1} \bar{t}_{\lambda}^{-1}}}\left(\chi_{\rho_{k}}^{\dagger} \circ N_{K_{n} / K_{k}}\right)(\mathfrak{a}) \psi^{\dagger}\left(\xi_{1} \tilde{t}_{\lambda}^{-1}\right) \\
& \times \sum_{\substack{\xi_{2}=\tilde{c} \tilde{c}, c \in \mathcal{F}_{n}, b \in \bar{t}_{\lambda},}}\left(\left(\operatorname{det} \rho_{k}\right)^{\dagger} \circ N_{F_{n} / F_{k}}\right)^{-1}(\tilde{c}) \psi^{\dagger}(\tilde{c})^{-2}
\end{aligned}
$$

One defines an algebraic-valued distribution on $\mathcal{G}_{n}$ by

$$
\int_{\mathcal{G}_{n}} \psi \cdot \mathrm{d} \mu_{E, \rho}:=\frac{(-4 i)^{\left[F_{n}: \mathbb{Q}\right]}}{N_{F_{n} / \mathbb{Q}}\left(\mathfrak{d}_{F}\right) \alpha\left(\mathfrak{m}^{\prime} \mathfrak{l}^{\prime}\right) C\left(\mathfrak{c}\left(\mathbf{f}_{E}\right), \mathbf{f}_{E}\right)} \mathcal{L}_{F_{n}}\left(\Phi_{\psi}^{n, k} \mid U\left(\mathfrak{m}^{\prime} \mathfrak{l}^{\prime} \mathfrak{r}_{0}^{-1}\right)\right)
$$

with respect to the finitely additive functions $\psi$. Note that the above proposition implies the right-hand side is independent of the choice of ideals $\mathfrak{m}^{\prime}$ and $\mathfrak{l}^{\prime}$. 
Let us now specialise to the situation of the Introduction. Recall our elliptic curve $E$ was semistable over $F_{n}$ with bad multiplicative reduction at $\mathfrak{p}$, so that $\alpha(\mathfrak{p})=a_{p}(E)$ whilst $\alpha^{\prime}(\mathfrak{p})=0$. The Euler factor in the above proposition at the primes dividing $\mathfrak{l}_{0}$ can be shown to equal 1, via the same argument as was outlined in [7, proof of Lemma 3.5]. It follows that

$$
\operatorname{Eul}_{\mathfrak{p l}}\left(\rho_{k} / F_{n} \otimes \psi^{-1}, s\right)=\left(1-a_{p}(E) \beta(\mathfrak{p}) \psi^{-1}(\mathfrak{p}) p^{s-1}\right)\left(1-a_{p}(E) \beta^{\prime}(\mathfrak{p}) \psi^{-1}(\mathfrak{p}) p^{s-1}\right) .
$$

The latter factor always equals 1 unless $\psi$ is the trivial character and $\operatorname{Res}_{K_{n}}\left(\chi_{\rho_{k}}\right)(\mathfrak{P})=1$ where $\mathfrak{P}$ is the unique prime of $K_{n}$ above $\mathfrak{p}$, in which case

$$
\operatorname{Eul}_{\mathfrak{p l} \mathfrak{l}_{0}}\left(\rho_{k} / F_{n}, s\right)=1-a_{p}(E) p^{s-1} .
$$

The above term vanishes at $s=1$ when $a_{p}(E)=+1$ (this causes the trivial zero in $\mathbf{L}_{p}\left(E, \rho_{k} / F_{n}\right)$ for small values of $\left.k\right)$.

Remark: Assume $\psi$ is ramified only at the prime above $p$. Applying an identical argument to [7, proof of Theorem 3.2], one obtains the following formula linking the HMF $\Phi_{\psi}^{n, k}$ with Artin-twists of the Hasse-Weil $L$-function of $E_{/ F_{n}}$ :

$$
\begin{aligned}
\frac{i^{h_{F_{n}}}(-4 i)^{\phi\left(p^{n}\right) / 2}}{\alpha\left(\mathfrak{m}^{\prime} \mathfrak{l}^{\prime}\right) C\left(\mathfrak{c}\left(\mathbf{f}_{E}\right), \mathbf{f}_{E}\right)} \quad \mathcal{L}_{F_{n}}\left(\Phi_{\psi}^{n, k} \mid U\left(\mathfrak{m}^{\prime} \mathfrak{l}^{\prime} \mathfrak{l}_{0}^{-1}\right)\right) & =\frac{\epsilon_{F_{n}}\left(\rho_{k} / F_{n} \otimes \psi\right)}{a_{p}(E)^{f\left(\rho_{k} / F_{n} \otimes \psi, \mathfrak{p}\right)} \prod_{q \mid \Delta} \alpha_{q}^{\phi\left(p^{n}\right)}} \\
\times \quad \operatorname{Eul}_{\mathfrak{p l}_{0}}\left(\rho_{k} / F_{n} \otimes \psi^{-1}, 1\right) & \times \frac{L_{S}\left(E, \rho_{k} / F_{n} \otimes \psi^{-1}, 1\right)}{\left(\Omega_{E}^{+} \Omega_{E}^{-}\right)^{\left[F_{n}: \mathbb{Q}\right]}}
\end{aligned}
$$

where $h_{F_{n}}$ denoted the narrow class number of $F_{n}$.

\subsection{Proof of Theorems 1 and 2}

We will now prove that after embedding $\tau_{p}: \overline{\mathbb{Q}} \hookrightarrow \overline{\mathbb{Q}}_{p}$, we obtain a bounded $p$-adic measure. Let $\psi$ be a character of $\mathcal{G}_{n}=\operatorname{Gal}\left(F_{n, S}^{\mathrm{ab}} / F_{n}\right)$; we define for integers $n \geq k$ the integral

$$
\int_{x \in \mathcal{G}_{n}} \psi(x) \mathrm{d} \mu_{E, p}^{n, k}(x):=\tau_{p}\left(\gamma_{E}^{n, k} \times \frac{i^{h_{F_{n}}}(-4 i)^{\phi\left(p^{n}\right) / 2}}{\alpha\left(\mathfrak{m}^{\prime} \mathfrak{l}^{\prime}\right) C\left(\mathfrak{c}\left(\mathbf{f}_{E}\right), \mathbf{f}_{E}\right)} \times \mathcal{L}_{F_{n}}\left(\Phi_{\psi}^{n, k} \mid U\left(\mathfrak{m}^{\prime} \mathfrak{l}^{\prime} \mathfrak{l}_{0}^{-1}\right)\right)\right)
$$

where $\gamma_{E}^{n, k}:=\frac{\prod_{q \mid \Delta} \alpha_{q}^{\phi\left(p^{n}\right)}}{\prod_{\nu \neq \mathfrak{p}} \epsilon_{F_{n}}\left(\rho_{k} / F_{n}\right)_{\nu}}$, and the Hilbert modular form

$$
\begin{aligned}
\Phi_{\psi}^{n, k} & =\Phi^{n, k}\left(\rho_{k} / F_{n} \otimes \psi, \mathfrak{c}\left(\mathbf{f}_{E}\right) \mathfrak{m}^{\prime} \mathfrak{l}^{\prime}\right) \\
& =\left(\mathbf{g}_{\rho_{k} / F_{n} \otimes \psi}\right) \times E_{1}\left(0, \mathfrak{c}\left(\mathbf{f}_{E}\right) \mathfrak{m}^{\prime} \mathfrak{l}^{\prime},\left(\operatorname{Res}_{F_{n}} \operatorname{det} \rho_{k}\right)^{\dagger-1} \otimes \psi^{\dagger-2}\right)
\end{aligned}
$$

was as in the previous section.

Proposition 18. (i) The above distribution $\mu_{E, p}^{n, k}$ yields a p-bounded measure on $\mathcal{G}_{n}$.

(ii) For characters $\psi$ on $\mathcal{G}_{n}$ of $\mathfrak{p}$-power conductor, the special values of $d \mu_{E, p}^{n, k}(x)$ at $\psi$ equal

$\int_{x \in \mathcal{G}_{n}} \psi(x) d \mu_{E, p}^{n, k}(x)=\frac{\epsilon_{F_{n}}\left(\rho_{k} / F_{n} \otimes \psi\right)_{\mathfrak{p}}}{a_{p}(E)^{f\left(\rho_{k} / F_{n} \otimes \psi, \mathfrak{p}\right)}} \times \operatorname{Eul}_{\mathfrak{p l}_{0}}\left(\rho_{k} / F_{n} \otimes \psi^{-1}, 1\right) \times \frac{L_{S}\left(E, \rho_{k} / F_{n} \otimes \psi^{-1}, 1\right)}{\left(\Omega_{E}^{+} \Omega_{E}^{-}\right)^{\left[F_{n}: \mathbb{Q}\right]}}$.

(iii) The corresponding power series $\mathbf{L}_{p}\left(E, \rho_{k} / F_{n}, T\right)=\int_{x \in \mathbb{Z}_{p}}(1+T)^{p^{n-1} x} \mathrm{~d} \mu_{E, p}^{n, k}(x)$ belongs to the algebra $\mathbb{Z}_{p}\left[\left[(1+T)^{p^{n-1}}-1\right]\right] \otimes \mathbb{Q} \cong \mathbb{Z}_{p}\left[\left[U^{(n)}\right]\right] \otimes \mathbb{Q}$. 
Proof. To show that $\mu_{E, p}^{n, k}$ is bounded, it suffices to check the Kummer congruences - the argument is the same as [7, proof of Proposition 3.3], which proves (i). Part (ii) follows from (6) and (7). Finally (iii) is a consequence of [3, Theorem 4.2] which implies that all special values $\tau_{p}^{-1}\left(\psi\left(\mathbf{L}_{p}\left(E, \rho_{k} / F_{n}\right)\right)\right)$ are $\operatorname{Aut}(\mathbb{C})$-equivariant.

In particular, choosing $k=n$ in parts (ii),(iii) above yields Theorem 1 as a consequence.

We now explain the link between $N_{k, n}\left(\mathbf{L}_{p}\left(E, \rho_{k} / F_{k}\right)\right)$ and $\mathbf{L}_{p}\left(E, \rho_{k} / F_{n}\right)$, where $N_{k, n}$ is the norm map $\mathbb{Z}_{p}\left[\left[U^{(k)}\right]\right] \rightarrow \mathbb{Z}_{p}\left[\left[U^{(n)}\right]\right]$ for $n \geq k$. Let $\psi: \mathcal{G}_{k} \rightarrow \mathbb{C}^{\times}$be a multiplicative character; by Artin formalism, there is an equality of complex $L$-functions

$$
\prod_{\eta: \operatorname{Gal}\left(F_{n} / F_{k}\right) \rightarrow \mathbb{C}^{\times}} L\left(E, \rho_{k} / F_{k} \otimes(\eta \psi)^{-1}, s\right)=L\left(E, \rho_{k} / F_{n} \otimes\left(\operatorname{Res}_{F_{n}} \psi\right)^{-1}, s\right)
$$

and those characters $\eta$ in the product may be identified with characters on $U^{(k)} / U^{(n)}$. Similarly the inductivity of $\epsilon$-factors, conductors, Euler factors and the motivic periods (via the Artin formalism) implies that analogous base-change relations hold for the other terms in the interpolation formula; it follows that

$$
\prod_{\eta: U^{(k)} / U^{(n)} \rightarrow \overline{\mathbb{Q}}_{p}^{\times}} \eta \psi\left(\mathbf{L}_{p}\left(E, \rho_{k} / F_{k}\right)\right)=\int \operatorname{Res}_{U^{(n)}}(\psi) \cdot d \mu_{E, p}^{n, k}=\psi\left(\mathbf{L}_{p}\left(E, \rho_{k} / F_{n}\right)\right)
$$

at every character $\psi: U^{(k)} \rightarrow \overline{\mathbb{Q}}_{p}^{\times}$, whence $N_{k, n}\left(\mathbf{L}_{p}\left(E, \rho_{k} / F_{k}\right)\right)=\mathbf{L}_{p}\left(E, \rho_{k} / F_{n}\right)$ (for $k=0$, the element $\mathbf{L}_{p}\left(E, \rho_{0} / F_{n}\right)$ coincides with the norm of the $p$-adic $L$-function of [17]).

In order to prove Theorem 2, we must establish the system of $p$-adic congruences $\mathbf{L}_{p}\left(E, \rho_{k} / F_{n}\right) \equiv \mathbf{L}_{p}\left(E, \rho_{n} / F_{n}\right) \bmod p \mathbb{Z}_{p}\left[\left[U^{(n)}\right]\right]$ for all $n \geq k$. In fact after evaluating at each character $\psi$, it is sufficient to establish them modulo the maximal ideal of $\mathbb{C}_{p}$. This requires a detailed study of the Fourier expansion of the Hilbert modular form $\Phi_{\psi}^{n, k}$.

By Atkin-Lehner theory, the linear functional $\mathcal{L}_{F_{n}}$ decomposes into a finite linear combination of the Fourier coefficients, so there exist finitely many ideals $\mathfrak{n}_{i}$ and fixed algebraic numbers $l\left(\mathfrak{n}_{i}\right) \in \overline{\mathbb{Q}}$ such that

$$
\mathcal{L}_{F_{n}}(\Theta)=\sum_{i} C\left(\mathfrak{n}_{i}, \Theta\right) l\left(\mathfrak{n}_{i}\right)
$$

Therefore putting $u:=\frac{i^{h} F_{n}(-4 i)^{\phi\left(p^{n}\right) / 2}}{C(\mathfrak{c}(\mathbf{f}), \mathbf{f}) \alpha\left(\mathfrak{m}^{\prime} \mathfrak{l}^{\prime}\right)}$ which is a $p$-adic unit, we have

$$
\begin{aligned}
\sum_{\psi} B b_{\psi} \int_{x \in \mathcal{G}_{n}} \psi(x) d \mu(x) & =u B \sum_{\psi} b_{\psi} \mathcal{L}_{F_{n}}\left(\Phi_{\psi}^{n, k} \mid U\left(\mathfrak{m}^{\prime} \mathfrak{l}^{\prime} \mathfrak{l}_{0}^{-1}\right)\right) \\
& =u B \sum_{\psi} b_{\psi} \sum_{i} C\left(\mathfrak{n}_{i}, \Phi_{\psi}^{n, k} \mid U\left(\mathfrak{m}^{\prime} \mathfrak{l}^{\prime} \mathfrak{l}_{0}^{-1}\right)\right) l\left(\mathfrak{n}_{i}\right) \\
& =u \sum_{i}\left(\sum_{\psi} b_{\psi} C\left(\mathfrak{n}_{i} \mathfrak{m}^{\prime} \mathfrak{l}^{\prime} \mathfrak{l}_{0}^{-1}, \Phi_{\psi}^{n, k}\right)\right) B l\left(\mathfrak{n}_{i}\right)
\end{aligned}
$$

By the same reasoning as [7, proof of Lemma 3.5], it suffices to show the congruences

$$
C\left(\mathfrak{m}, \Phi_{\psi}^{n, k}\right) \equiv C\left(\mathfrak{m}, \Phi_{\psi}^{n, n}\right) \quad \bmod \mathfrak{M}_{\mathbb{C}_{p}}
$$

hold amongst the Fourier coefficients, for all $\mathfrak{m}$ and $\psi$. 
Fixing an ideal $\mathfrak{m}=\xi \tilde{t}_{\lambda}^{-1}$ and applying the second statement in Proposition 17, we have congruences

$$
\begin{aligned}
C\left(\mathfrak{m}, \Phi_{\psi}^{n, k}\right) & =\sum_{\xi_{1}+\xi_{2}=\xi} \sum_{\substack{\mathfrak{a} \triangleleft \mathcal{O}_{K_{n}}, \mathfrak{a} \overline{\mathfrak{a}}=\xi_{1} \bar{t}_{\lambda}^{-1}}}\left(\chi_{\rho_{k}}^{\dagger} \circ N_{K_{n} / K_{k}}\right)(\mathfrak{a}) \psi^{\dagger}(\mathfrak{a} \overline{\mathfrak{a}}) \sum_{\substack{\xi_{2}=\tilde{b} \tilde{c}, c \in \mathcal{O}_{n}, b \in \bar{t}_{\lambda}}}\left(\left(\operatorname{det} \rho_{k}\right)^{\dagger} \circ N_{F_{n} / F_{k}}\right)^{-1}(\tilde{c}) \psi^{\dagger}(\tilde{c})^{-2} \\
& \equiv \sum_{\xi_{1}, \xi_{2}} \sum_{\mathfrak{a}} \psi^{\dagger}(\mathfrak{a} \overline{\mathfrak{a}}) \sum_{c}\left(\theta_{K_{k} / F_{k}} \circ N_{F_{n} / F_{k}}\right)(\tilde{c}) \psi^{\dagger}(\tilde{c})^{-2} \bmod \mathfrak{M}_{\mathbb{C}_{p}},
\end{aligned}
$$

where $\theta_{K_{k} / F_{k}}$ is the quadratic character as defined in (2), and

$$
\begin{aligned}
C\left(\mathfrak{m}, \Phi_{\psi}^{n, n}\right) & =\sum_{\xi_{1}+\xi_{2}=\xi} \sum_{\substack{\mathfrak{a} \triangleleft \mathcal{O}_{K_{n}}, \mathfrak{a} \overline{\mathfrak{a}}=\xi_{1} \tilde{t}_{\lambda}^{-1}}} \chi_{\rho_{n}}^{\dagger}(\mathfrak{a}) \psi^{\dagger}(\mathfrak{a} \overline{\mathfrak{a}}) \sum_{\substack{\xi_{2}=\tilde{b} \tilde{c}, c \in \mathcal{O}, b \in \bar{t}_{n},}}\left(\operatorname{det} \rho_{k}\right)^{\dagger-1}(\tilde{c}) \psi^{\dagger}(\tilde{c})^{-2} \\
& \equiv \sum_{\xi_{1}, \xi_{2}} \sum_{\mathfrak{a}} \psi^{\dagger}(\mathfrak{a} \overline{\mathfrak{a}}) \sum_{c} \theta_{K_{n} / F_{n}}(\tilde{c}) \psi^{\dagger}(\tilde{c})^{-2} \quad \bmod \mathfrak{M}_{\mathbb{C}_{p} .}
\end{aligned}
$$

Lastly since $\theta_{K_{k} / F_{k}} \circ N_{F_{n} / F_{k}}=\theta_{K_{n} / F_{n}}$ the congruences (8) are immediately established, and Theorem 2 is proved.

\subsection{Proof of Theorem 4}

From our discussion directly after Proposition 17, the Euler factor in the interpolation formula for $\mathbf{L}_{p}\left(E, \rho_{k} / F_{n}\right)$ is given by

$$
\operatorname{Eul}_{\mathfrak{p l}_{0}}\left(\rho_{k} / F_{n} \otimes \psi^{-1}, s\right)=\left(1-a_{p}(E) \operatorname{Res}_{K_{n}}\left(\chi_{\rho_{k}}\right)(\mathfrak{P}) \psi^{-1}(\mathfrak{p}) p^{s-1}\right)
$$

which vanishes at the critical point $s=1$ if and only if $a_{p}(E)=+1, \psi$ is the trivial character, and $\operatorname{Res}_{K_{n}}\left(\chi_{\rho_{k}}\right)(\mathfrak{P})=1$.

Lemma 19. If $\delta=\operatorname{ord}_{p}\left(\Delta^{p-1}-1\right)-1$ then

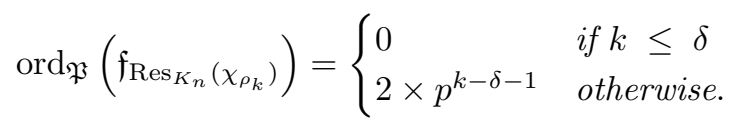

Proof. We decompose $\Delta$ into $c \times(1+d)$ where $c \in \mu_{p-1}$ and $d \in p \mathbb{Z}_{p}$, thus $\delta+1=\operatorname{ord}_{p}(d)$. Recall that if $x, y \in \mathbb{Q}_{p}\left(\mu_{p^{n}}\right)$, the $p^{n}$-th norm residue symbol $(x, y)$ is the $p^{n}$-th root of unity satisfying

$$
\theta(y)\left(\sqrt[p^{n}]{x}\right)=(x, y) \sqrt[p^{n}]{x}
$$

where $\theta$ is the local Artin map $\mathbb{Q}_{p}\left(\mu_{p^{n}}\right)^{\times} /\left(\mathbb{Q}_{p}\left(\mu_{p^{n}}\right)^{\times}\right)^{p^{n}} \longrightarrow \operatorname{Gal}\left(\mathbb{Q}_{p}\left(\mu_{p^{n}}, \sqrt[p^{n}]{x}\right) / \mathbb{Q}_{p}\left(\mu_{p^{n}}\right)\right)$. Indeed we have

$$
\operatorname{Res}_{K_{n}}\left(\chi_{\rho_{k}}\right)(\sigma)=\frac{\sigma\left(\sqrt[p^{k}]{\Delta}\right)}{\sqrt[p^{k}]{\Delta}}=\frac{\sigma\left(\sqrt[p^{n}]{\Delta^{p^{n-k}}}\right)}{\sqrt[p^{n}]{\Delta^{p^{n-k}}}}
$$

so locally, the character $\operatorname{Res}_{K_{n}}\left(\chi_{\rho_{k}}\right)$ is simply the $p^{n}$-th norm residue symbol $\left(\Delta^{p^{n-k}},-\right)$. But $\Delta^{p^{n-k}}=c^{p^{n-k}} \times\left(1+d_{n, k}\right)$ for some $d_{n, k} \in p \mathbb{Z}_{p}$, therefore by [5] (also [22, Theorem 8])

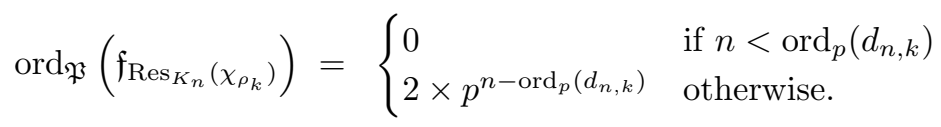


However $1+d_{n, k}=(1+d)^{p^{n-k}}$ and $\operatorname{ord}_{p}\left((1+d)^{p^{n-k}}-1\right)=n-k+\operatorname{ord}_{p}(d)=n-k+\delta+1$, which produces the stated formula.

Without loss of generality, we assume that $E$ has split multiplicative reduction, so that $a_{p}(E)=+1$. Then the first statement in Theorem 4 follows immediately, as $\operatorname{Eul}_{\mathfrak{p r}}\left(\rho_{n}, 1\right)=$ 0 precisely when $\chi_{\rho_{n}}$ has trivial $\mathfrak{P}$-conductor, which by the above lemma occurs if and only if $n \leq \delta$. To establish Theorem 4(ii), we use the decomposition

$$
\mathbf{L}_{p}\left(E / \mathbb{Q}\left(\sqrt[p^{n}]{\Delta}\right), T\right)=\mathbf{L}_{p}\left(E, \omega^{0}, T\right) \times \prod_{j=1}^{n} \mathbf{L}_{p}\left(E, \rho_{j}, T\right)
$$

which yields a trivial zero contribution of order $1+\sum_{j=1}^{\min (\delta, n)} 1=\min (\delta+1, n+1)$. Moreover

$$
p \cdot \mathcal{O}_{\mathbb{Q}\left(\sqrt[p^{n}]{\Delta}\right)}= \begin{cases}\mathcal{P}_{(n), 0} \mathcal{P}_{(n), 1}^{p-1} \mathcal{P}_{(n), 2}^{p(p-1)} \ldots \mathcal{P}_{(n), n}^{p^{n-1}(p-1)} & \text { if } n \leq \delta \\ \left(\mathcal{P}_{(n), 0} \mathcal{P}_{(n), 1}^{p-1} \mathcal{P}_{(n), 2}^{p(p-1)} \ldots \mathcal{P}_{(n), \delta}^{p^{\delta-1}(p-1)}\right)^{p^{n-\delta}} & \text { if } n>\delta\end{cases}
$$

for distinct prime ideals $\mathcal{P}_{(n), j} \in \operatorname{Spec}\left(\mathcal{O}_{\mathbb{Q}\left(\sqrt[p^{n}]{\Delta}\right)}\right)$ by $[16$, Lemma 2.2]; hence $\min (\delta+1, n+1)$ coincides with the number of primes ideals lying above $p$, namely $\delta_{n}$ as asserted.

\section{$2.4 \lambda$-invariants and the Proof of Theorem 6}

Let $\lambda^{\text {an }}\left(E, \rho_{n}\right)$ be the number of zeros of $\mathbf{L}_{p}\left(E, \rho_{n}\right)$ counted with multiplicity, where we view the latter as an element of $\mathbb{Z}_{p}\left[\left[U^{(1)}\right]\right] \cong \mathbb{Z}_{p}[[T]]$ via the inclusion $U^{(n)} \hookrightarrow U^{(1)}$. Likewise $\lambda^{\text {an }}\left(E, \omega^{j}\right)$ is the $\lambda$-invariant of the $\omega^{j}$-th branch of the Mazur-Tate-Teitelbaum $p$-adic $L$-function $\mathbf{L}_{p}\left(E, \omega^{j}\right)$ from [17].

Proposition 20. If we assume Hypothesis $(\mu=0)$, then for all integers $n \geq 1$ :

(a) There is a linear growth formula $\lambda^{\text {an }}\left(E, \rho_{n}\right)=p^{n-1} \times \sum_{j=0}^{p-2} \lambda^{\text {an }}\left(E, \omega^{j}\right)$;

(b) Over each $L_{n}=\mathbb{Q}\left(\mu_{p^{n}}, \sqrt[p^{n}]{\Delta}\right)$, one has $\lambda_{L_{n}}^{\mathrm{an}}(E)=p^{2 n-1} \times \sum_{j=0}^{p-2} \lambda^{\mathrm{an}}\left(E, \omega^{j}\right)$.

Proof. Applying [6, Lemma 2.1], the congruence in Theorem 2 implies that the elements on both sides share the same $\lambda$-invariant. Now by definition,

$$
N_{0, n}\left(a_{0}\right)=N_{1, n}\left(\prod_{j=0}^{p-2} \mathbf{L}_{p}\left(E, \omega^{j}\right)\right)
$$

and if $\mathcal{F} \in \mathbb{Z}_{p}\left[\left[U^{(1)}\right]\right]$ then the $\lambda$-invariant of $N_{1, n}(\mathcal{F})$ equals that of $\mathcal{F}$ multiplied by $p^{n-1}$, therefore (a) follows directly. To establish part (b), we know that

$$
\lambda_{L_{n}}^{\mathrm{an}}(E)=\sum_{\rho \in \operatorname{Irr}\left(\operatorname{Gal}\left(L_{n} / \mathbb{Q}\right)\right)} \operatorname{deg}(\rho) \times \lambda^{\mathrm{an}}(E, \rho) \quad \text { from Equation (1). }
$$

However, one can decompose the irreducible representations into the disjoint union $\operatorname{Irr}\left(\operatorname{Gal}\left(L_{n} / \mathbb{Q}\right)\right)=\left\{\theta: \operatorname{Gal}\left(K_{n} / \mathbb{Q}\right) \rightarrow \overline{\mathbb{Q}}^{\times}\right\} \cup\left\{\rho_{t} \otimes \theta \mid 1 \leq t \leq n, \theta: \operatorname{Gal}\left(K_{n} / K_{t}\right) \rightarrow \overline{\mathbb{Q}}^{\times}\right\}$ so the result follows from (a), together with some direct calculations. 
Remarks: If the curve $E$ has split multiplicative reduction at $p$, then the right-hand side of Proposition 20(a) will be positive because of the trivial zero in $\mathbf{L}_{p}\left(E, \omega^{0}\right)$, hence one must have $\lambda^{\text {an }}\left(E, \rho_{n}\right)>0$; however when $n>\delta$, the corresponding $\rho_{n}$-twisted $p$ adic $L$-function does not satisfy the trivial zero condition. If it were then the case that $\operatorname{corank}_{\mathbb{Z}_{p}}\left(\operatorname{Sel}_{p \infty}\left(E / L_{n}\right)^{\rho_{n}}\right)>0$, this would suggest (assuming the BSD conjecture) the vanishing of $L\left(E, \rho_{n}, s\right)$ at $s=1$ for integers $n>\delta$, even if the root number in the complex $\rho_{n}$-twisted $L$-function is +1 .

We now explain how to deduce Theorem 6 from the previous proposition. Firstly

$$
p \cdot \mathcal{O}_{L_{n}}= \begin{cases}\prod_{i=1}^{p^{n}} \mathfrak{p}_{(n), i}^{p^{n-1}(p-1)} & \text { if } n \leq \delta \\ \prod_{i=1}^{p^{\delta}} \mathfrak{p}_{(n), i}^{p^{2 n-\delta-1}(p-1)} & \text { if } n>\delta\end{cases}
$$

by [16, Proposition 2.4], so there are precisely $p^{\min (\delta, n)}$ prime ideals of $\mathcal{O}_{L_{n}}$ lying above $p$. Therefore one concludes

$$
\begin{aligned}
& r_{L_{n}}^{\dagger}(E)=\operatorname{order}_{T=0}\left(\mathbf{L}_{p}\left(E / L_{n}, T\right)\right)-\#\left\{\text { places } \nu \text { of } L_{n} \text { over } p\right\} \times \frac{1+a_{p}(E)}{2} \\
& \leq\left[U^{(1)}: U^{(n)}\right]^{-1} \times \lambda_{L_{n}}^{\text {an }}(E)-p^{\min (\delta, n)} \times \frac{1+a_{p}(E)}{2} \\
& \text { by } \stackrel{20(\mathrm{~b})}{=} p^{1-n} \times\left(p^{2 n-1} \times \sum_{j=0}^{p-2} \lambda^{\text {an }}\left(E, \omega^{j}\right)\right)-p^{\min (\delta, n)} \times \frac{1+a_{p}(E)}{2}
\end{aligned}
$$

and Theorem 6(i) follows as a consequence.

To prove the second statement, observe that

$$
\begin{aligned}
& r_{\mathbb{Q}\left(p^{n} \sqrt{\Delta}\right)}^{\dagger}(E)+\left(\frac{1+a_{p}(E)}{2}\right) \times \delta_{n}=\operatorname{order}_{T=0}\left(\mathbf{L}_{p}\left(E / \mathbb{Q}\left(\sqrt[p^{n}]{\Delta}\right), T\right)\right) \\
& =\operatorname{order}_{T=0}\left(\mathbf{L}_{p}\left(E, \omega^{0}, T\right)\right)+\sum_{j=1}^{n} \operatorname{order}_{T=0}\left(\mathbf{L}_{p}\left(E, \rho_{j}, T\right)\right) \\
& \leq \lambda_{\mathbb{Q}}^{\mathrm{an}}(E)+\sum_{j=1}^{n} \lambda^{\mathrm{an}}\left(E, \rho_{j}\right) \stackrel{\text { by }}{=}{ }^{20(\mathrm{a})} \lambda_{\mathbb{Q}}^{\mathrm{an}}(E)+\sum_{j=1}^{n} p^{j-1} \times \lambda_{\mathbb{Q}\left(\mu_{p}\right)}^{\mathrm{an}}(E)
\end{aligned}
$$

and summing up the geometric progression, clearly Theorem 6(ii) must also hold true.

\section{The Algebraic Side}

We now study the behaviour of the Selmer group of $E$ over $\mathbb{Q}_{F T}$. Throughout we fix a number field $K=L_{n, m}=\mathbb{Q}\left(\mu_{p^{n}}, \sqrt[p^{m}]{\Delta}\right)$ for integers $n \geq m>0$, so that $K / \mathbb{Q}$ is a finite Galois extension contained inside $\mathbb{Q}_{F T}$. As in the Introduction, one assumes $M=\operatorname{Sel}_{p \infty}\left(E / \mathbb{Q}_{F T}\right)^{\wedge}$ belongs to the $\mathfrak{M}_{\mathcal{H}}\left(G_{\infty}\right)$-category, and denotes by reg ${ }_{K}$ the regular representation of $\operatorname{Gal}(K / \mathbb{Q})$. 


\subsection{Leading terms of characteristic elements}

We begin with some background on the evaluation-at- $\rho$ map $\Phi_{\rho}^{\prime}$. Let $\mathcal{O}$ be the ring of integers of some finite extension of $\mathbb{Q}_{p}$, and set $\Gamma:=\operatorname{Gal}\left(\mathbb{Q}^{\text {cyc }} / \mathbb{Q}\right)$ so that $G_{\infty} / \mathcal{H} \cong \Gamma$. One writes $\rho^{\dagger}: \mathbb{Z}_{p}\left[\left[G_{\infty}\right]\right] \longrightarrow \operatorname{Mat}_{n \times n}(\mathcal{O})$ for the ring homomorphism induced from an Artin representation $\rho: G_{\infty} \rightarrow \operatorname{GL}(n, \mathcal{O})$.

The continuous group homomorphism $G_{\infty} \longrightarrow$ Mat $_{n \times n}(\mathcal{O}[[\Gamma]])$ that sends $g \in G_{\infty}$ to $\rho^{\dagger}(g) \otimes(g \bmod \mathcal{H})$ extends to a localised algebra homomorphism

$$
\Phi_{\rho}: \mathbb{Z}_{p}\left[\left[G_{\infty}\right]\right]_{\mathcal{S}^{*}} \longrightarrow \operatorname{Mat}_{n \times n}\left(Q_{\mathcal{O}}(\Gamma)\right)
$$

where $Q_{\mathcal{O}}(\Gamma)$ indicates the skew-field of quotients of $\mathcal{O}[[\Gamma]]$ (see $[4$, Lemma 3.3] for details). On the level of K-groups, we then have a unique extension

$$
\Phi_{\rho}^{\prime}: \mathrm{K}_{1}\left(\mathbb{Z}_{p}\left[\left[G_{\infty}\right]\right]_{\mathcal{S}^{*}}\right) \longrightarrow \mathrm{K}_{1}\left(\operatorname{Mat}_{n \times n}\left(Q_{\mathcal{O}}(\Gamma)\right)\right) \cong Q_{\mathcal{O}}(\Gamma)^{\times}
$$

where the last isomorphism arises by Morita invariance.

Recall from the Introduction that if $M$ is an object lying in the category $\mathfrak{M}_{\mathcal{H}}\left(G_{\infty}\right)$, we wrote $\xi_{M}$ for a characteristic element in $\mathrm{K}_{1}\left(\mathbb{Z}_{p}\left[\left[G_{\infty}\right]\right]_{\mathcal{S}^{*}}\right)$ satisfying $\partial_{G_{\infty}}\left(\xi_{M}\right)=[M]$. For each Artin representation $\rho: G_{\mathbb{Q}} \rightarrow G_{\infty} \rightarrow \mathrm{GL}(V)$, by using [4, Diagram (43)] one has the Akashi series relation

$$
\Phi_{\rho}^{\prime}\left(\xi_{M}\right) \equiv \prod_{j \geq 0} \operatorname{char}_{\mathbb{Z}_{p}[[\Gamma]]}\left(H_{j}\left(\mathcal{H}, \operatorname{tw}_{\rho}(M)\right)\right)^{(-1)^{j}} \bmod \mathbb{Z}_{p}[[\Gamma]]^{\times}
$$

We write $K^{\text {cyc }}$ for the cyclotomic $\mathbb{Z}_{p}$-extension of $K$, and $\Gamma_{K}$ will denote its Galois group (we fix a topological generator $\gamma_{K}$ of $\left.\Gamma_{K}\right)$. Define $\mathcal{H}_{K}:=\operatorname{Gal}\left(\mathbb{Q}_{F T} / K^{\text {cyc }}\right)$ ) so that there is an inclusion $\mathcal{H}_{K} \hookrightarrow \mathcal{H}$; in particular, plugging in $\rho=\operatorname{reg}_{K}$ one obtains an isomorphism

$$
H_{j}\left(\mathcal{H}, \operatorname{tw}_{\rho}(M)\right) \cong H_{j}\left(\mathcal{H}_{K}, M\right) \otimes \operatorname{reg}_{\Gamma / \Gamma_{K}}
$$

via Shapiro's lemma. Moreover from [16, proof of 5.4], we have $H_{j}\left(\mathcal{H}_{K}, M\right)=0$ for all indices $j \geq 1$, in which case

$$
\left.\Phi_{\operatorname{reg}_{K}}^{\prime}\left(\xi_{M}\right) \equiv \operatorname{char}_{\mathbb{Z}_{p}\left[\left[\Gamma_{K}\right]\right]}\left(H_{0}\left(\mathcal{H}_{K}, M\right)\right)\right) \bmod \mathbb{Z}_{p}\left[\left[\Gamma_{K}\right]\right]^{\times}
$$

Suppose that $\kappa: G_{\mathbb{Q}} \rightarrow \operatorname{Gal}\left(\mathbb{Q}\left(\mu_{p^{\infty}}\right) / \mathbb{Q}\right) \stackrel{\sim}{\rightarrow} \mathbb{Z}_{p}^{\times}$denotes the $p$-adic cyclotomic character.

Lemma 21. (i) If $E$ has split multplicative reduction at $p$, then

$$
\begin{aligned}
\Phi_{\operatorname{reg}_{K}}^{\prime}\left(\xi_{M}\right) \equiv \prod_{\nu \mid \Delta} \operatorname{char}_{\mathbb{Z}_{p}\left[\left[\Gamma_{K}\right]\right]}\left(J_{\nu}\left(K^{\mathrm{cyc}}\right)^{\wedge}\right) \times \prod_{v_{n} \mid p}\left((T+1)^{p^{n-1}}-\kappa\left(\gamma_{v_{n}}\right)\right) \\
\quad \times \operatorname{char}_{\mathbb{Z}_{p}\left[\left[\Gamma_{K}\right]\right]}\left(\operatorname{Sel}_{p}\left(E / K^{\mathrm{cyc}}\right)^{\wedge}\right) \bmod \mathbb{Z}_{p}\left[\left[\Gamma_{K}\right]\right]^{\times}
\end{aligned}
$$

where $\gamma_{v_{n}}$ denotes a topological generator of the decomposition group at a place of $K^{\text {cyc }}$ lying above $v_{n}$, and $J_{\nu}\left(K^{\mathrm{cyc}}\right)=\lim _{K^{\prime} \subset K^{\mathrm{cyc}}} \bigoplus_{\pi \mid \nu} H^{1}\left(K_{\pi}^{\prime}, E\right)\left[p^{\infty}\right]$.

(ii) If E has non-split multplicative reduction at p, then the same formula holds but without the $\prod_{v_{n} \mid p}\left((T+1)^{p^{n-1}}-\kappa\left(\gamma_{v_{n}}\right)\right)$-term appearing. 
Proof. This is essentially [27, Theorem 6.2]. Indeed the proof of Theorem 1.3 in op. cit. implies that

$\Phi_{\operatorname{reg}_{K}}^{\prime}\left(\xi_{M}\right) \equiv \operatorname{char}\left(\operatorname{Sel}_{p^{\infty}}\left(E / K^{\mathrm{cyc}}\right)^{\wedge}\right) \times \prod_{\nu \mid \Delta} \operatorname{char}\left(J_{\nu}\left(K^{\mathrm{cyc}}\right)^{\wedge}\right) \times \prod_{v_{n} \mid p} \operatorname{char}\left(H^{1}\left(\mathcal{H}_{w}, D_{w}\right)^{\wedge}\right)$

modulo $\mathbb{Z}_{p}\left[\left[\Gamma_{K}\right]\right]^{\times}$, where $D_{w}=\mathbb{Q}_{p} / \mathbb{Z}_{p}$ if the eigenvalue $a_{p}(E)=+1$, and $D_{w}=\mathbb{Q}_{p} / \mathbb{Z}_{p} \otimes \theta$ for an unramified quadratic character $\theta$ if $a_{p}(E)=-1$.

Note that $\mathcal{H}_{w} \cong \mathbb{Z}_{p} \rtimes \Gamma_{x}$ where the $\mathbb{Z}_{p}$-extension $\Gamma_{x}$ acts on $\mathbb{Z}_{p}$ through $\kappa$, and $x$ is some place of $K^{\text {cyc }}$ above $v$. In particular $H^{j}\left(\mathcal{H}_{w}, \mathbb{Q}_{p} / \mathbb{Z}_{p}\right)=0$ for every $j \geq 2$ by applying a cohomological dimension argument. Moreover $H^{1}\left(\mathcal{H}_{w}, D_{w}\right)$ is finite if $a_{p}(E)=-1$, hence its dual has unit characteristic power series, which then proves statement (ii) above. Alternatively, if we assume $a_{p}(E)=+1$ then $H^{1}\left(\mathcal{H}_{w}, D_{w}\right)^{\wedge}=H^{1}\left(\mathcal{H}_{w}, \mathbb{Q}_{p} / \mathbb{Z}_{p}\right)^{\wedge} \cong \mathbb{Z}_{p}(\kappa)$ as a local $\Lambda$-module, and (i) follows immediately.

We now analyse the term $\prod_{v_{n} \mid p}\left((T+1)^{p^{n-1}}-\kappa\left(\gamma_{v_{n}}\right)\right)$ in the split multiplicative case. Recalling that $K=L_{n, m}$, the cyclotomic character yields an isomorphism

$$
\left(\kappa \bmod G_{K^{\mathrm{cyc}}}\right): \operatorname{Gal}\left(K^{\mathrm{cyc}} / \mathbb{Q}\left(\mu_{p}, \sqrt[p^{m}]{\Delta}\right)\right) \stackrel{\sim}{\longrightarrow} 1+p \mathbb{Z}_{p}
$$

Moreover each decomposition group at a place of $K^{\mathrm{cyc}}$ over $v_{n} \in \operatorname{Spec}\left(\mathcal{O}_{K}\right)$ is isomorphic to $1+p^{n} \mathbb{Z}_{p}$ via $\kappa$, in which case

$$
\prod_{v_{n} \mid p}\left((T+1)^{p^{n-1}}-\kappa\left(\gamma_{v_{n}}\right)\right)=\prod_{v_{n} \mid p}\left((T+1)^{p^{n-1}}-\kappa\left(u_{1}^{p^{n-1}}\right)\right)=\left((T+1)^{p^{n-1}}-\kappa\left(u_{1}\right)^{p^{n-1}}\right)^{\#\left\{v_{n} \mid p\right\}}
$$

where $u_{1}$ denotes a topological generator of $U^{(1)}$.

If $n=m$ then as we have already seen, there are exactly $p^{\min (\delta, m)}$ places $v_{n}$ above $p$. However if $n>m$ then there are $p^{\min (\delta, m)}$ places of $L_{m}$ over $p$, and the field extension $L_{n, m} / L_{m}$ is totally ramified at each of these places, so again there must be $p^{\min (\delta, m)}$ places of $K=L_{n, m}$ lying over $p$. We have therefore shown

Corollary 22. The term $\prod_{v_{n} \mid p}\left((T+1)^{p^{n-1}}-\kappa\left(\gamma_{v_{n}}\right)\right)$ equals $\left((T+1)^{p^{n-1}}-\kappa\left(u_{1}\right)^{p^{n-1}}\right)^{p^{\min (\delta, m)}}$.

We can directly apply these two results to relate the leading term of $\Phi_{\operatorname{reg}_{K}}^{\prime}\left(\xi_{M}\right)$ with the cyclotomic Selmer group. Firstly the power series $\operatorname{char}\left(J_{\nu}\left(K^{\mathrm{cyc}}\right)^{\wedge}\right)$ has non-zero constant term, equal to the $L$-factor $L_{\nu}(E, 1) \bmod \mathbb{Z}_{p}^{\times}$by [26, Lemma 2.14] and [27, Remark 1.4]. Moreover $(T+1)^{p^{n-1}}-\left.\kappa\left(u_{1}\right)^{p^{n-1}}\right|_{T=0}$ belongs to $p^{n} \mathbb{Z}_{p}$ but not $p^{n+1} \mathbb{Z}_{p}$, so the product term in the previous corollary contributes $p^{n p^{\min (\delta, m)}} \bmod \mathbb{Z}_{p}^{\times}$.

Corollary 23. The power series $\Phi_{\mathrm{reg}_{K}}^{\prime}\left(\xi_{M}\right)$ has the same order of vanishing at $T=0$ as the characteristic power series of $\operatorname{Sel}_{p \infty}\left(E / K^{\mathrm{cyc}}\right)^{\wedge}$, and their leading terms differ by

$$
\nabla_{\Gamma_{K}}^{G_{\infty}}(E):=\left\{\begin{aligned}
p^{n p^{\min (\delta, m)}} \times \prod_{\nu \mid \Delta} L_{\nu}(E, 1) & \bmod \mathbb{Z}_{p}^{\times} & \text {if } a_{p}(E)=+1 \\
\prod_{\nu \mid \Delta} L_{\nu}(E, 1) & \bmod \mathbb{Z}_{p}^{\times} & \text {if } a_{p}(E)=-1 .
\end{aligned}\right.
$$




\subsection{Proof of Theorems 7 and 9}

Let $\mathcal{M}=\operatorname{Hom}_{\mathrm{cts}}\left(\operatorname{Sel}_{p^{\infty}}\left(E / K^{\text {cyc }}\right), \mathbb{Q} / \mathbb{Z}\right)$ denote the Pontryagin dual of the Selmer group over the cyclotomic $\mathbb{Z}_{p}$-extension of $K$, and set $\mathcal{L}_{\mathcal{M}}=\operatorname{char}_{\mathbb{Z}_{p}\left[\left[\Gamma_{K}\right]\right]}(\mathcal{M})$ which is well defined up to an element of $\mathbb{Z}_{p}\left[\left[\Gamma_{K}\right]\right]^{\times}$. There exists a sequence of homomorphisms

$$
H^{0}\left(\Gamma_{K}, \mathcal{M}\right)=\mathcal{M}^{\Gamma_{K}} \hookrightarrow \mathcal{M} \rightarrow \mathcal{M} /\left(\gamma_{K}-1\right) \cong H^{1}\left(\Gamma_{K}, \mathcal{M}\right)
$$

and we shall denote their composition by $\alpha_{\mathcal{M}}$. To simplify the exposition, assume that:

(i) the $p$-primary part of the Tate-Shafarevich group $\mathbf{I I I}(E / K)$ for $E$ over $K$ is finite;

(ii) the $p$-adic height pairing $\langle-,-\rangle_{K}: E(K) \times E(K) \rightarrow \mathbb{Q}_{p}$ is non-degenerate $[14,19]$.

Theorem 24. (Jones [14, Theorem 3.1])

(a) If the natural mapping $\alpha_{\mathcal{M}}: H^{0}\left(\Gamma_{K}, \mathcal{M}\right) \rightarrow H^{1}\left(\Gamma_{K}, \mathcal{M}\right)$ is not a quasi-isomorphism then $r_{\mathcal{M}}:=\operatorname{order}_{T=0}\left(\operatorname{char}_{\mathbb{Z}_{p}[[T]]}(\mathcal{M})\right)>\operatorname{rank}_{\mathbb{Z}_{p}}\left(\mathcal{M}^{\Gamma_{K}}\right)$.

(b) If the natural mapping $H^{0}\left(\Gamma_{K}, \mathcal{M}\right) \rightarrow H^{1}\left(\Gamma_{K}, \mathcal{M}\right)$ is a quasi-isomorphism then $\operatorname{order}_{T=0}\left(\operatorname{char}_{\mathbb{Z}_{p}[[T]]}(\mathcal{M})\right)=\operatorname{rank}_{\mathbb{Z}_{p}}\left(\mathcal{M}^{\Gamma_{K}}\right)$, with the higher derivative formula

$$
\begin{aligned}
\frac{1}{r_{\mathcal{M}} !} \cdot \frac{\mathrm{d}^{r_{\mathcal{M}}} \mathcal{L}_{\mathcal{M}}\left(\kappa\left(\gamma_{K}\right)^{1-s}-1\right)}{\mathrm{d} s^{r_{\mathcal{M}}}} & \equiv \ell_{p}(E) \times \# \mathbf{I I I}(E / K)_{p^{\infty}} \times \operatorname{det}\left(\langle-,-\rangle_{K}\right) \\
& \times \prod_{\nu \nmid \infty}\left[E\left(K_{\nu}\right): E_{0}\left(K_{\nu}\right)\right] \times \# E(K)_{\text {tors }}^{-2} \bmod \mathbb{Z}_{p}^{\times} .
\end{aligned}
$$

The quantity $\operatorname{rank}_{\mathbb{Z}_{p}}\left(\mathcal{M}^{\Gamma_{K}}\right)$ coincides with $s_{n, m}(E)$ in the Introduction when $K=L_{n, m}$, because the restriction map $\operatorname{Sel}_{p}(E / K) \rightarrow \operatorname{Sel}_{p}\left(E / K^{\mathrm{cyc}}\right)^{\Gamma_{K}}$ is quasi-injective, and its

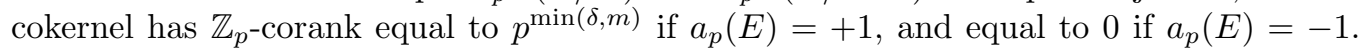
Furthermore there are inequalities

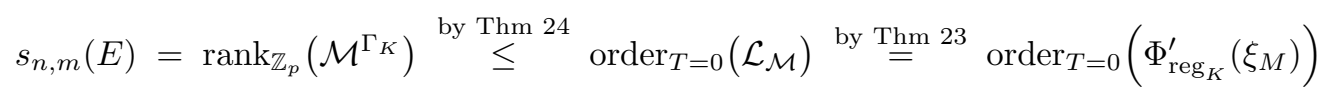

which are all bounded above by $\lambda_{K}^{\text {alg }}(E)$, and therefore Theorem $7(\mathrm{a})$ is proved.

Combining the previous theorem with Corollary 23, then Theorem 7 (b) follows as a direct consequence of the congruence

$$
\frac{1}{r_{\mathcal{M}} !} \cdot \frac{\mathrm{d}^{r_{\mathcal{M}}} \Phi_{\mathrm{reg}_{K}}^{\prime}\left(\xi_{M}\right)}{\mathrm{d} T^{r_{\mathcal{M}}}} \equiv \nabla_{\Gamma_{K}}^{G_{\infty}}(E) \times \frac{1}{r_{\mathcal{M}} !} \cdot \frac{\mathrm{d}^{r_{\mathcal{M}}} \mathcal{L}_{\mathcal{M}}\left(\kappa\left(\gamma_{K}\right)^{1-s}-1\right)}{\mathrm{d} s^{r_{\mathcal{M}}}} \quad \bmod \mathbb{Z}_{p}^{\times}
$$

Theorem 9 is now immediate as the finiteness of $\operatorname{Sel}_{p} \infty(E / K)$ implies both the finiteness of the $p$-primary part of $\mathbf{I I I}(E / K)$, and the non-degeneracy of the $p$-adic height pairing (indeed $E(K) \otimes \mathbb{Q}_{p}$ will be zero, so this pairing does not even appear in the calculation of the leading term). 


\subsection{Formulating the Iwasawa Main Conjecture}

The statement of our conjecture follows the format of Coates et al [4] in the $\mathrm{GL}_{2}$-setting, and in the non-split case there are essentially no surprises. Intriguingly in the case of split multiplicative reduction, the specialisations $\Phi_{\rho_{n}}^{\prime}\left(\xi_{M}\right)$ are divisible by an extra factor $\mathcal{D}_{p}\left(\rho_{n}, T\right)$ when $n \leq \delta$, and so the Main Conjecture would imply the same divisibility holds for the analytic $p$-adic $L$-function $\mathbf{L}_{p}\left(E, \rho_{n}, T\right)$ if $n \leq \delta$. Lloyd Peters' numerical calculations in the Appendix support this divisibility for some sample elliptic curves $E / \mathbb{Q}$.

Definition 25. (a) If $\sigma: G_{\infty} \rightarrow \mathrm{GL}(V)$ is an irreducible Artin representation, then

$$
\mathcal{D}_{p}(\sigma, T):= \begin{cases}T+1-\sigma \kappa\left(u_{1}\right) & \text { if } \operatorname{dim}(\sigma)=1 \text { with }\left.\sigma\right|_{U_{\text {tors }}^{(0)}}=\mathbf{1} \\ (T+1)^{p^{n-1}}-\left(\psi \kappa\left(u_{1}\right)\right)^{p^{n-1}} & \text { if } \sigma \cong \rho_{n} \otimes \psi \text { with } 1 \leq n \leq \delta \\ 1 & \text { otherwise. }\end{cases}
$$

(b) If $\rho \cong \bigoplus_{\sigma \in \operatorname{Irr}\left(G_{\infty}\right)} \sigma^{\oplus e_{\sigma}}$ then one defines $\mathcal{D}_{p}(\rho, T):=\prod_{\sigma \in \operatorname{Irr}\left(G_{\infty}\right)} \mathcal{D}_{p}(\sigma, T)^{e_{\sigma}}$.

The motivation for introducing these $\mathcal{D}_{p}$-factors is that, when specialised to the regular representation (at any finite layer in $\mathbb{Q}_{F T}$ ), they coincide with the terms in Corollary 22.

Proposition 26. If $\rho=\operatorname{reg}_{K}$ where the number field $K=L_{n, m} \neq \mathbb{Q}$, then

$$
\mathcal{D}_{p}\left(\operatorname{reg}_{K}, T\right)=\left((T+1)^{p^{n-1}}-\kappa\left(u_{1}\right)^{p^{n-1}}\right)^{p^{\min (\delta, m)}}
$$

and consequently $\mathcal{D}_{p}\left(\operatorname{reg}_{K}, 0\right) \equiv p^{n p^{\min (\delta, m)}} \bmod \mathbb{Z}_{p}^{\times}$.

Proof. We proceed by induction on both $n, m$. Let us treat the diagonal case $n=m$ first. If $n=1$ then $\operatorname{reg}_{L_{1}} \cong \bigoplus_{j=0}^{p-2} \omega^{j} \oplus \rho_{1}^{p-1}$, in which case

$\mathcal{D}_{p}\left(\operatorname{reg}_{L_{1}}, T\right)=\left(T+1-\kappa\left(u_{1}\right)\right) \times\left\{\begin{array}{ll}\left(T+1-\kappa\left(u_{1}\right)\right)^{p-1} & \text { if } \delta>0 \\ 1 & \text { if } \delta=0\end{array}\right\}=\left(T+1-\kappa\left(u_{1}\right)\right)^{p^{\min (\delta, 1)}}$.

Assume further that $n>1$, and the predicted formula for $\mathcal{D}_{p}\left(\operatorname{reg}_{L_{n-1}}, T\right)$ above is correct. It is easy to see that

$$
\operatorname{reg}_{L_{n}} \cong\left(\bigoplus_{\psi} \operatorname{reg}_{L_{n-1}} \otimes \psi\right) \oplus \rho_{n}^{p^{n}-p^{n-1}}
$$

where the sum runs over any $p$ characters $\psi: \operatorname{Gal}\left(K_{n} / \mathbb{Q}\right) \rightarrow \mathbb{C}^{\times}$whose restrictions to $\operatorname{Gal}\left(K_{n} / K_{n-1}\right)$ are pairwise distinct (in fact, one can even assume $\left.\psi\right|_{U_{\text {tors }}^{(0)}}=\mathbf{1}$ for each $\psi$ ).

Case(I): If $n \leq \delta$ then

$$
\begin{aligned}
& \mathcal{D}_{p}\left(\operatorname{reg}_{L_{n}}, T\right)=\prod_{\psi} \mathcal{D}_{p}\left(\operatorname{reg}_{L_{n-1}} \otimes \psi, T\right) \times \mathcal{D}_{p}\left(\rho_{n}, T\right)^{p^{n}-p^{n-1}} \\
& \quad=\prod_{\psi}\left((T+1)^{p^{n-2}}-\left(\psi \kappa\left(u_{1}\right)\right)^{p^{n-2}}\right)^{p^{n-1}} \times \mathcal{D}_{p}\left(\rho_{n}, T\right)^{p^{n}-p^{n-1}} \\
& \quad=\prod_{\zeta \in \mu_{p}}\left((T+1)^{p^{n-2}}-\zeta \times \kappa\left(u_{1}\right)^{p^{n-2}}\right)^{p^{n-1}} \times\left((T+1)^{p^{n-1}}-\kappa\left(u_{1}\right)^{p^{n-1}}\right)^{p^{n}-p^{n-1}} \\
& =\left((T+1)^{p^{n-1}}-\kappa\left(u_{1}\right)^{p^{n-1}}\right)^{p^{n-1}+p^{n}-p^{n-1}}=\left((T+1)^{p^{n-1}}-\kappa\left(u_{1}\right)^{p^{n-1}}\right)^{p^{\min (\delta, n)}} .
\end{aligned}
$$


Case(II): If $n>\delta$ then

$$
\begin{aligned}
\mathcal{D}_{p}\left(\operatorname{reg}_{L_{n}}, T\right) & =\prod_{\psi} \mathcal{D}_{p}\left(\operatorname{reg}_{L_{n-1}} \otimes \psi, T\right) \times \mathcal{D}_{p}\left(\rho_{n}, T\right)^{p^{n}-p^{n-1}} \\
& =\prod_{\psi}\left((T+1)^{p^{n-2}}-\left(\psi \kappa\left(u_{1}\right)\right)^{p^{n-2}}\right)^{p^{\delta}} \times 1^{p^{n}-p^{n-1}} \\
& =\prod_{\zeta \in \mu_{p}}\left((T+1)^{p^{n-2}}-\zeta \times \kappa\left(u_{1}\right)^{p^{n-2}}\right)^{p^{\delta}}=\left((T+1)^{p^{n-1}}-\kappa\left(u_{1}\right)^{p^{n-1}}\right)^{p^{\min (\delta, n)}} .
\end{aligned}
$$

To extend to the non-diagonal situation $n>m>0$, recall that $\operatorname{reg}_{L_{n, m}} \cong \bigoplus_{\theta} \operatorname{reg}_{L_{m}} \otimes \theta$ where the $p^{n-m}$ characters $\theta: \operatorname{Gal}\left(K_{n} / \mathbb{Q}\right) \rightarrow \mathbb{C}^{\times}$are pairwise distinct on $\operatorname{Gal}\left(K_{n} / K_{m}\right)$. Therefore, since the diagonal case is already established, we deduce

$$
\begin{aligned}
& \mathcal{D}_{p}\left(\operatorname{reg}_{L_{n, m}}, T\right)=\prod_{\theta} \mathcal{D}_{p}\left(\operatorname{reg}_{L_{m}} \otimes \theta, T\right)=\prod_{\theta}\left((T+1)^{p^{m-1}}-\left(\theta \kappa\left(u_{1}\right)\right)^{p^{m-1}}\right)^{p^{\min (\delta, m)}} \\
& =\prod_{\zeta \in \mu_{p^{n-m}}}\left((T+1)^{p^{m-1}}-\zeta \times \kappa\left(u_{1}\right)^{p^{m-1}}\right)^{p^{\min (\delta, m)}}=\left((T+1)^{p^{n-1}}-\kappa\left(u_{1}\right)^{p^{n-1}}\right)^{p^{\min (\delta, m)}}
\end{aligned}
$$

as required.

Let $\iota: \mathrm{K}_{1}\left(\mathbb{Z}_{p}\left[\left[G_{\infty}\right]\right]\right) \longrightarrow \mathrm{K}_{1}\left(\mathbb{Z}_{p}\left[\left[G_{\infty}\right]\right]_{\mathcal{S}^{*}}\right)$ denote the homomorphism induced from the natural map $\mathbb{Z}_{p}\left[\left[G_{\infty}\right]\right] \longrightarrow \mathbb{Z}_{p}\left[\left[G_{\infty}\right]\right]_{\mathcal{S}^{*}}$ into the localisation. We can relate the abelian $p$-adic $L$-functions constructed in Theorem 1 with the Selmer group over $\mathbb{Q}_{F T}$, as follows.

Conjecture 27 (Main Conjecture). There exists an element $\mathfrak{u} \in \mathrm{K}_{1}\left(\mathbb{Z}_{p}\left[\left[G_{\infty}\right]\right]\right)$ satisfying

$$
\Phi_{\rho}^{\prime}\left(\xi_{M}\right)=\Phi_{\rho}^{\prime}(\iota(\mathfrak{u})) \times \mathbf{L}_{p}\left(E, \rho, u_{1}-1\right)
$$

at every Artin representation $\rho: G_{\mathbb{Q}} \rightarrow G_{\infty} \rightarrow \mathrm{GL}(V)$.

The term $\mathfrak{u}$ arises as there are many choices of lift $\xi_{M}$ for the class $[M]$ under the projection $\partial_{G_{\infty}}: K_{1}\left(\mathbb{Z}_{p}\left[\left[G_{\infty}\right]\right]_{\mathcal{S}^{*}}\right) \rightarrow K_{0}\left(\mathfrak{M}_{\mathcal{H}}\left(G_{\infty}\right)\right)$. Thus the Main Conjecture predicts $\xi_{M} / \iota(\mathfrak{u})$ is the canonical choice of algebraic element, compatible with the $L$-functions in Theorem 1.

We conclude the main text by focussing solely on the split multiplicative case $a_{p}(E)=+1$. The discussion at the start of this section indicates that $\Phi_{\rho}^{\prime}\left(\xi_{M}\right)$ should be divisible by $\mathcal{D}_{p}(\rho, T)$, and so the same should therefore be true for $\mathbf{L}_{p}(E, \rho, T)$ via the Main Conjecture. Let us now consider the representation $\operatorname{Ind}_{K_{n}}^{\mathbb{Q}}(\mathbf{1}) \cong \bigoplus_{\psi \in \operatorname{reg}_{K_{n}}} \psi$.

Remark: If the prime $p \geq 5$, then a straightforward application of the Greenberg-Stevens formula [13, Theorem 7.1] implies that

$$
\left.\frac{\mathrm{d} \mathbf{L}_{p}\left(E, \operatorname{Ind}_{K_{n}}^{\mathbb{Q}}(\mathbf{1}), T\right)}{\mathrm{d} T}\right|_{T=0}=\frac{\log _{p}\left(q_{E, p}\right)}{\operatorname{ord}_{p}\left(q_{E, p}\right)} \times \mathcal{L}_{E}\left(\operatorname{Ind}_{K_{n}}^{\mathbb{Q}}(\mathbf{1})\right)
$$

where the algebraic $L$-value $\mathcal{L}_{E}\left(\operatorname{Ind}_{K_{n}}^{\mathbb{Q}}(\mathbf{1})\right):=\sqrt{\operatorname{disc}_{K_{n}}} \times \frac{L_{S}\left(E / K_{n}, 1\right)}{\left(\Omega_{E}^{+} \Omega_{E}^{-}\right)^{\left[F_{n}: \mathbb{Q}\right]}}$ 
As a consequence, one has $\mathbf{L}_{p}\left(E / K_{n}, T\right)=0+\frac{\log _{p}\left(q_{E, p}\right)}{\operatorname{ord}_{p}\left(q_{E, p}\right)} \times \mathcal{L}_{E}\left(\operatorname{Ind}_{K_{n}}^{\mathbb{Q}}(\mathbf{1})\right) \times T+O\left(T^{2}\right)$. If $n=1$ then $\mathcal{D}_{p}\left(\operatorname{reg}_{K_{1}}, T\right)=T-\left(\kappa\left(u_{1}\right)-1\right)=p w+T+O\left(T^{2}\right)$ for some unit $w \in \mathbb{Z}_{p}^{\times}$; it follows that $\mathcal{D}_{p}\left(\operatorname{reg}_{K_{1}}, T\right)$ can only divide into $\mathbf{L}_{p}\left(E / K_{1}, T\right)$, provided the derivative of $\mathbf{L}_{p}\left(E / K_{1}, T\right)$ lies in the maximal ideal of $\mathbb{Z}_{p}$.

Conjecture 28. If $a_{p}(E)=+1$ then $\frac{\log _{p}\left(q_{E, p}\right)}{\operatorname{ord}_{p}\left(q_{E, p}\right)} \times \mathcal{L}_{E}\left(\operatorname{reg}_{K_{1}}\right) \in p \mathbb{Z}_{p}$.

In the Appendix below, for the primes $p=3$ and 5 it is verified that $\mathcal{L}_{E}\left(\operatorname{reg}_{\mathbb{Q}\left(\mu_{p}\right)}\right) \in p \mathbb{Z}_{p}$ at various choices of $\Delta \leq 97$, and for a selection of elliptic curves $E$ of conductor $\leq 70$

Acknowledgements: Many ideas in this paper were developed during the second named author's visit in New Zealand in May 2013, which was supported by an ARC-DP1092496 grant. He would like to thank the Maths Department at the University of Waikato for the warm hospitality that he received, and the administrative staff of Monash University for the organisation of his trip. His research is currently supported by FRQNT's Établissement de nouveaux chercheurs universitaires program 188809. Finally the two authors would like to acknowledge Tom Ward for allowing the use of his MAGMA code, and thank Lloyd Peters for undertaking the delicate computer calculations appearing in both the Introduction and the Appendix.

\section{References}

[1] K. Barré-Sirieix, G. Diaz, F. Gramain, G. Philibert: Une preuve de la conjecture de Mahler-Manin, Invent. Math. 124 (1996), no. 1-3, p 1-9.

[2] A. Bouganis: Special values of L-functions and false Tate curve extensions, Journal of the London Math. Soc. 82 (2010), p 596-620.

[3] A. Bouganis, V. Dokchitser: Algebraicity of L-values for elliptic curves in a false Tate curve tower, Math. Proc. Camb. Phil. Soc. 142 (2007), no. 2, p 193-204.

[4] J. Coates, T. Fukaya, K. Kato, R. Sujatha, O. Venjakob: The $G L_{2}$ main conjecture for elliptic curves without complex multiplication, Publ. Math. IHES 101 (2005), p 163-208.

[5] R. Coleman, W. McCallum: Stable reduction of Fermat curves and Jacobi sum Hecke characters, J. Reine Angew. Math. 385, (1988), p 41-101.

[6] D. Delbourgo, A. Lei: Transition formulae for ranks of abelian varieties, to appear in Rocky Mountain Math. Journal (2015).

[7] D. Delbourgo, T. Ward: Non-abelian congruences between L-values of elliptic curves, Ann. Inst. Fourier (Grenoble) 58 (2008), no. 3, p 10231055. 
[8] D. Delbourgo, T. Ward: The growth of CM periods over false Tate extensions, Experiment. Math. 19 (2010), no. 2, p 195-210.

[9] D. Delbourgo: Exceptional zeroes of p-adic L-functions over non-abelian field extensions, to appear in Glasgow Math. Journal (2015).

[10] D. Delbourgo: On trivial p-adic zeroes for elliptic curves over Kummer extensions, New Zealand Journal of Mathematics 45 (2015), p 33-38.

[11] T. Dokchitser, V. Dokchitser: Computations in non-commutative Iwasawa theory, Proc. London Math. Soc. (3) 94 (2007), p 211-272.

[12] V. Dokchitser: Root numbers of non-abelian twists of elliptic curves, Proc. London Math. Soc. (3) 91 (2005), p 300-324.

[13] R. Greenberg, G. Stevens: p-adic L-functions and p-adic periods of modular forms, Invent. Math. 111 (1993), p 401-447.

[14] J. W. Jones: Iwasawa L-functions for multiplicative abelian varieties, Duke Math. J. 59 (1989), no. 2, p 399-420.

[15] K. Kato: $K_{1}$ of some non-commutative completed group rings, $K$-theory 34 (2005), no. 2, p 99-140.

[16] C.-Y. Lee: Non-commutative Iwasawa theory of elliptic curves at primes of multiplicative reduction, Math. Proc. Cambridge Philos. Soc. 154 (2013), no. 2, p 303-324.

[17] B. Mazur, J. Tate, J. Teitelbaum: On p-adic analogues of the conjectures of Birch and Swinnerton-Dyer, Invent. Math. 84 (1986), no. 1, 1-48.

[18] A. A. Panchishkin: Non-Archimedean L-functions of Siegel and Hilbert modular forms, Springer-Verlag, Lecture Notes in Mathematics vol 1471 (1991).

[19] P. Schneider: p-adic height pairings. I., Invent. Math. 69 (1982), no. 3, p 401-409.

[20] P. Schneider: p-adic height pairings. II. , Invent. Math. 79 (1985), no. 2, p 329-374.

[21] J.-P. Serre: Sur les représentations modulaires de degré 2 de $\operatorname{Gal}(\overline{\mathbb{Q}} / \mathbb{Q})$, Duke Math. J. 54 (1987), no. 1, p 179-230.

[22] R. Sharifi: On norm residue symbols and conductors, J. Number Theory, 86 (2001), no. 2, p 196-209. 
[23] G. Shimura: Special values of the zeta functions associated with Hilbert modular forms, Duke Math. J. 45 (1978), no. 3, p 637-679.

[24] J. Tate: Number theoretic background, Proceedings of Symposia in Pure Mathematics, 33 (1979), (2) p 3-26.

[25] F. Viviani: Ramification groups and Artin conductors of radical extensions of $\mathbb{Q}, \mathrm{J}$. Théor. Nombres Bordeaux 16 (2004), no. 3, p 779-816.

[26] S. L. Zerbes: Generalised Euler characteristics of Selmer groups, Proc. Lond. Math. Soc. 98 (2009), no. 3, p 775-96.

[27] S. L. Zerbes: Akashi series of Selmer groups, Math. Proc. Cambridge Philos. Soc. 151 (2011), no. 2, p 229-243. 


\title{
Appendix - Numerical Calculations at 3 and 5
}

\author{
by Lloyd Christopher Peters
}

Our aim is to numerically verify the first layer $\bmod p$ congruences predicted in this paper. Due to computational limitations, the congruences are only checked at the primes 3 and 5 . The author thanks Tom Ward for providing him with his personal code, and the University of Sydney for granting him a free MAGMA developer's licence.

The notations employed here are almost identical to the previous text; in particular $E_{/ \mathbb{Q}}$ denotes an elliptic curve with bad multiplicative reduction at a fixed odd prime $p$. Let us now define $K:=\mathbb{Q}\left(\mu_{p}\right), F:=\mathbb{Q}\left(\mu_{p}\right)^{+}$and $L:=\mathbb{Q}\left(\mu_{p}, \sqrt[p]{\Delta}\right)$.

Remark: We write $\sigma$ for the regular representation of $\operatorname{Gal}(K / \mathbb{Q})$, and $\rho$ for the self-dual irreducible Artin representation on $\operatorname{Gal}(L / \mathbb{Q})$ of dimension $p-1$. Note that $\sigma$ decomposes into the sum $\bigoplus_{i=1}^{p-1} \omega^{i}$, which means the $\sigma$-twisted Hasse-Weil $L$-function becomes

$$
L(E, \sigma, s)=\prod_{i=1}^{p-1} L\left(E, \omega^{i}, s\right)
$$

The right hand side of this equation is easier to compute (at $s=1$ ) than the left hand side since $\omega^{i}$ is 1-dimensional; moreover on the level of $\epsilon$-factors, we have $\epsilon_{F}(\sigma)_{p}=\prod_{i=1}^{p-1} \epsilon\left(\omega^{i}\right)_{p}$.

Recall that $S$ denoted the set of primes dividing $\Delta$, and $\delta:=\operatorname{ord}_{p}\left(\Delta^{p-1}-1\right)-1 \geq 0$. Henceforth we shall treat only examples where $\delta=0$. Consider the following quantities:

- $L^{*}=\left|\frac{L(E, \rho, 1) \sqrt{\operatorname{disc}_{F}}}{\left(2 \Omega_{E}^{+} \Omega_{E}^{-}\right)^{\frac{(p-1)}{2}}}\right|$ where $\operatorname{disc}_{F}$ denotes the discriminant of $F$.

- $\mathbf{1}\left(\mathbf{L}_{p}(E, \rho)\right)=\frac{L_{S}(E, \rho, 1)}{\left(\Omega_{E}^{+} \Omega_{E}^{-}\right)^{\frac{p-1}{2}}} \cdot \frac{\epsilon_{F}(\rho)_{p}}{a_{p}(E)^{f(\rho, p)}}\left(1-a_{p}(E) \chi_{\rho}(\mathfrak{P})\right)$ with $\mathfrak{P}$ the prime above $p$; in fact, the condition $\delta=0$ ensures that $\left(1-a_{p}(E) \chi_{\rho}(\mathfrak{P})\right)=1$.

- $\mathbf{1}\left(\mathbf{L}_{p}(E, \sigma)\right)=\frac{L_{S}(E, \sigma, 1)}{\left(\Omega_{E}^{+} \Omega_{E}^{-}\right)^{\frac{p-1}{2}}} \cdot \frac{\epsilon_{F}(\sigma)_{p}}{a_{p}(E)^{f(\sigma, p)}} \cdot\left(1-a_{p}(E)\right)$.

If $a_{p}(E)=+1$ then $\left(1-a_{p}(E)\right)=0$, which produces an exceptional zero in $\mathbf{L}_{p}(E, \sigma, T)$. Therefore if $E$ has split multiplicative reduction, we instead tabulate the quantity

$$
\frac{\log _{p}\left(q_{E, p}\right)}{\operatorname{ord}_{p}\left(q_{E, p}\right)} \times \mathcal{L}_{E}\left(\operatorname{Ind}_{K}^{\mathbb{Q}}(\mathbf{1})\right)=\frac{\log _{p}\left(q_{E, p}\right)}{\operatorname{ord}_{p}\left(q_{E, p}\right)} \times \sqrt{\operatorname{disc}_{K}} \times \frac{L_{S}(E, \sigma, 1)}{\left(\Omega_{E}^{+} \Omega_{E}^{-}\right)^{\frac{p-1}{2}}}
$$

corresponding to the first derivative of the $p$-adic $L$-function attached to the $\sigma$-twist of $E$.

Throughout we have calculated $L(E, \sigma, 1), L(E, \rho, 1), P_{q}\left(E, \rho, q^{-1}\right), P_{q}\left(E, \sigma, q^{-1}\right)$, $\epsilon_{F}(\rho)_{p}, \epsilon_{F}(\sigma)_{p}, \Omega_{E}^{+}$and $\Omega_{E}^{-}$as complex numbers accurate to 15 digit decimal precision. The corresponding algebraic $L$-values are recorded in Tables $1-9$ by listing the coefficients of their $p$-adic expansions up to order $O\left(p^{8}\right)$.

To form a part of the author's Monash PhD thesis 
Table 1: E15a1 with equation $y^{2}+x y+y=x^{3}+x^{2}-10 x-10$, which has non-split multiplicative reduction at $p=3$.

\begin{tabular}{cccc}
\hline$\Delta$ & $L^{*}$ & $\mathbf{1}\left(\mathbf{L}_{p}(E, \rho)\right)$ & $\mathbf{1}\left(\mathbf{L}_{p}(E, \sigma)\right)$ \\
\hline 2 & 4 & {$[1,1,0,0,0,0,0,0,0]$} & {$[1,0,0,0,0,0,0,0,0]$} \\
5 & 0 & {$[0,0,0,0,0,0,0,0,0]$} & {$[0,1,2,2,1,2,2,0,0]$} \\
7 & 16 & {$[2,0,2,2,0,1,0,2,2]$} & {$[2,2,2,2,0,2,1,1,2]$} \\
11 & 16 & {$[1,2,2,0,0,1,1,2,2]$} & {$[1,2,0,1,1,0,2,0,0]$} \\
13 & 4 & {$[2,0,1,1,0,1,1,0,0]$} & {$[2,2,2,1,2,1,2,2,2]$} \\
14 & 4 & {$[1,2,1,2,0,1,0,2,2]$} & {$[1,2,2,2,1,1,0,0,0]$} \\
20 & 0 & {$[0,0,0,0,0,0,0,0,0]$} & {$[0,2,1,2,0,2,2,1,2]$} \\
22 & 64 & {$[2,1,2,1,0,2,2,1,2]$} & {$[2,1,1,2,2,0,1,1,0]$} \\
23 & 0 & {$[0,0,0,0,0,0,0,0,0]$} & {$[0,0,2,0,0,1,0,0,0]$} \\
29 & 4 & {$[1,1,0,1,2,2,0,0,0]$} & {$[1,1,0,0,0,0,2,2,2]$} \\
31 & 4 & {$[2,0,0,1,1,1,0,1,0]$} & {$[2,1,0,0,1,1,2,1,2]$} \\
34 & 64 & {$[2,2,2,2,1,2,0,2,2]$} & {$[2,1,1,0,2,2,2,2,2]$} \\
38 & 4 & {$[1,1,1,0,0,2,2,1,2]$} & {$[1,1,0,1,0,0,0,1,0]$} \\
41 & 4 & {$[1,2,1,0,2,0,1,2,2]$} & {$[1,2,0,0,0,0,0,1,0]$} \\
43 & 64 & {$[2,2,0,1,1,1,0,2,2]$} & {$[2,0,2,1,2,0,2,1,2]$} \\
47 & 16 & {$[1,2,0,2,2,1,0,1,0]$} & {$[1,0,2,2,0,1,1,1,0]$} \\
50 & 0 & {$[0,0,0,0,0,0,0,0,0]$} & {$[0,2,1,2,0,2,2,1,2]$} \\
52 & 100 & {$[1,2,1,2,2,0,2,2,2]$} & {$[1,2,2,0,2,0,2,2,2]$} \\
58 & 400 & {$[2,0,1,0,2,1,1,0,0]$} & {$[2,2,0,0,0,0,1,2,2]$} \\
59 & 64 & {$[1,1,0,1,0,1,1,0,0]$} & {$[1,1,2,2,2,0,0,0,0]$} \\
61 & 64 & {$[2,2,2,0,0,0,1,0,0]$} & {$[2,2,1,1,2,1,0,2,2]$} \\
67 & 16 & {$[2,2,1,1,0,0,2,2,2]$} & {$[2,2,0,0,2,1,0,2,2]$} \\
68 & 196 & {$[2,0,0,0,1,2,1,1,2]$} & {$[2,1,1,0,2,2,2,2,2]$} \\
70 & 0 & {$[0,0,0,0,0,0,0,0,0]$} & {$[0,2,2,0,1,2,2,2,2]$} \\
74 & 36 & {$[0,0,1,1,2,0,2,2,2]$} & {$[0,0,1,1,2,1,0,1,0]$} \\
76 & 64 & {$[1,0,2,1,2,2,0,0,0]$} & {$[1,1,0,1,0,0,0,1,0]$} \\
77 & 64 & {$[1,1,2,1,0,0,2,2,2]$} & {$[1,1,1,0,2,1,2,1,2]$} \\
79 & 64 & {$[2,2,1,0,0,1,2,1,2]$} & {$[2,2,1,2,2,2,2,2,2]$} \\
83 & 0 & {$[0,0,0,0,0,0,0,0,0]$} & {$[0,0,0,2,1,0,1,1,0]$} \\
85 & 0 & {$[0,0,0,0,0,0,0,0,0]$} & {$[0,2,2,2,2,2,1,1,2]$} \\
86 & 4 & {$[1,2,0,1,0,0,1,0,0]$} & {$[1,1,1,0,2,1,1,0,0]$} \\
92 & 36 & {$[0,0,2,2,2,1,0,2,2]$} & {$[0,0,1,1,0,2,0,0,0]$} \\
94 & 484 & {$[2,2,0,2,0,1,1,1,0]$} & {$[2,0,1,2,1,2,2,2,2]$} \\
95 & 0 & {$[0,0,0,0,0,0,0,0,0]$} & {$[0,1,0,2,2,0,2,2,2]$} \\
97 & 144 & {$[0,0,2,0,0,0,1,0,0]$} & {$[0,0,2,0,0,0,2,0,0]$} \\
\hline & & &
\end{tabular}


Table 2: E21a1 with Weierstrass equation $y^{2}+x y=x^{3}-4 x-1$, which has split multiplicative reduction at $p=3$.

\begin{tabular}{cccc}
\hline$\Delta$ & $L^{*}$ & $\mathbf{1}\left(\mathbf{L}_{p}(E, \rho)\right)$ & $\frac{\log _{p}\left(q_{E, p}\right)}{\operatorname{ord}_{p}\left(q_{E, p}\right)} \mathcal{L}_{E}\left(\operatorname{Ind}_{K}^{\mathbb{Q}}(\mathbf{1})\right)$ \\
\hline 2 & 0 & {$[0,0,0,0,0,0,0,0,0]$} & {$[0,2,2,2,2,0,1,0,0]$} \\
5 & 0 & {$[0,0,0,0,0,0,0,0,0]$} & {$[0,2,2,0,1,2,1,1,2]$} \\
7 & 0 & {$[0,0,0,0,0,0,0,0,0]$} & {$[0,1,0,0,0,1,2,0,0]$} \\
11 & 0 & {$[0,0,0,0,0,0,0,0,0]$} & {$[0,2,0,2,1,2,2,0,0]$} \\
13 & 0 & {$[0,0,0,0,0,0,0,0,0]$} & {$[0,1,0,0,1,2,2,2,2]$} \\
14 & 0 & {$[0,0,0,0,0,0,0,0,0]$} & {$[0,2,0,0,0,2,1,1,2]$} \\
20 & 0 & {$[0,0,0,0,0,0,0,0,0]$} & {$[0,1,2,1,2,1,0,0,0]$} \\
22 & 0 & {$[0,0,0,0,0,0,0,0,0]$} & {$[0,1,1,1,0,2,2,1,2]$} \\
23 & 0 & {$[0,0,0,0,0,0,0,0,0]$} & {$[0,0,0,1,2,2,1,1,2]$} \\
29 & 0 & {$[0,0,0,0,0,0,0,0,0]$} & {$[0,2,1,2,2,0,2,2,2]$} \\
31 & 0 & {$[0,0,0,0,0,0,0,0,0]$} & {$[0,1,1,2,2,0,2,2,2]$} \\
34 & 0 & {$[0,0,0,0,0,0,0,0,0]$} & {$[0,0,0,1,1,0,1,2,2]$} \\
38 & 0 & {$[0,0,0,0,0,0,0,0,0]$} & {$[0,2,1,2,1,0,2,1,2]$} \\
41 & 0 & {$[0,0,0,0,0,0,0,0,0]$} & {$[0,2,2,1,1,1,2,1,2]$} \\
43 & 0 & {$[0,0,0,0,0,0,0,0,0]$} & {$[0,0,0,1,1,1,2,1,2]$} \\
47 & 0 & {$[0,0,0,0,0,0,0,0,0]$} & {$[0,0,0,1,1,0,1,1,0]$} \\
50 & 0 & {$[0,0,0,0,0,0,0,0,0]$} & {$[0,1,2,1,2,1,0,0,0]$} \\
52 & 0 & {$[0,0,0,0,0,0,0,0,0]$} & {$[0,2,0,0,2,1,2,2,2]$} \\
58 & 0 & {$[0,0,0,0,0,0,0,0,0]$} & {$[0,1,0,2,2,1,1,2,2]$} \\
59 & 0 & {$[0,0,0,0,0,0,0,0,0]$} & {$[0,0,0,0,2,1,0,1,0]$} \\
61 & 0 & {$[0,0,0,0,0,0,0,0,0]$} & {$[0,1,0,1,1,2,2,0,0]$} \\
67 & 0 & {$[0,0,0,0,0,0,0,0,0]$} & {$[0,1,2,1,0,2,1,1,2]$} \\
68 & 0 & {$[0,0,0,0,0,0,0,0,0]$} & {$[0,0,0,1,1,0,1,2,2]$} \\
70 & 0 & {$[0,0,0,0,0,0,0,0,0]$} & {$[0,1,1,2,0,1,1,2,2]$} \\
74 & 0 & {$[0,0,0,0,0,0,0,0,0]$} & {$[0,2,0,2,1,1,1,0,0]$} \\
76 & 0 & {$[0,0,0,0,0,0,0,0,0]$} & {$[0,2,1,2,1,0,2,1,2]$} \\
77 & 0 & {$[0,0,0,0,0,0,0,0,0]$} & {$[0,2,1,1,2,1,0,0,0]$} \\
79 & 0 & {$[0,0,0,0,0,0,0,0,0]$} & {$[0,0,0,1,2,0,1,1,0]$} \\
83 & 0 & {$[0,0,0,0,0,0,0,0,0]$} & {$[0,0,0,0,1,1,2,1,2]$} \\
85 & 0 & {$[0,0,0,0,0,0,0,0,0]$} & {$[0,0,0,1,1,2,1,2,2]$} \\
86 & 0 & {$[0,0,0,0,0,0,0,0,0]$} & {$[0,0,0,2,2,2,1,0,0]$} \\
92 & 0 & {$[0,0,0,0,0,0,0,0,0]$} & {$[0,0,0,2,1,2,0,0,0]$} \\
94 & 0 & {$[0,0,0,0,0,0,0,0,0]$} & {$[0,0,0,2,2,0,2,2,2]$} \\
95 & 0 & {$[0,0,0,0,0,0,0,0,0]$} & {$[0,2,1,0,1,0,2,1,2]$} \\
\hline & 0 & {$[0,0,0,0,0,0,0,0,0]$} & {$[0,1,0,0,1,1,0,2,2]$} \\
\hline & & & \\
5 & &
\end{tabular}


Table 3: E30a1 with Weierstrass equation $y^{2}+x y+y=x^{3}+x+2$, which has split multiplicative reduction at $p=3$.

\begin{tabular}{cccc}
\hline$\Delta$ & $L^{*}$ & $\mathbf{1}\left(\mathbf{L}_{p}(E, \rho)\right)$ & $\frac{\log _{p}\left(q_{E, p}\right)}{\operatorname{ord}_{p}\left(q_{E, p}\right)} \mathcal{L}_{E}\left(\operatorname{Ind}_{K}^{\mathbb{Q}}(\mathbf{1})\right)$ \\
\hline 2 & 3 & {$[0,2,2,2,2,2,2,2,2]$} & {$[0,0,2,1,2,1,2,2,2]$} \\
5 & 12 & {$[0,2,0,2,1,0,1,2,2]$} & {$[0,0,1,0,1,2,1,2,2]$} \\
7 & 0 & {$[0,0,0,0,0,0,0,0,0]$} & {$[0,0,0,2,2,0,2,1,2]$} \\
11 & 0 & {$[0,0,0,0,0,0,0,0,0]$} & {$[0,0,0,2,2,2,2,0,0]$} \\
13 & 0 & {$[0,0,0,0,0,0,0,0,0]$} & {$[0,0,0,2,0,0,1,2,2]$} \\
14 & 27 & {$[0,0,0,2,1,2,0,1,0]$} & {$[0,0,0,0,2,0,0,2,2]$} \\
20 & 0 & {$[0,0,0,0,0,0,0,0,0]$} & {$[0,0,0,1,2,1,0,1,0]$} \\
22 & 0 & {$[0,0,0,0,0,0,0,0,0]$} & {$[0,0,0,0,2,0,2,0,0]$} \\
23 & 0 & {$[0,0,0,0,0,0,0,0,0]$} & {$[0,0,0,2,2,1,0,1,0]$} \\
29 & 0 & {$[0,0,0,0,0,0,0,0,0]$} & {$[0,0,0,0,1,2,0,1,0]$} \\
31 & 0 & {$[0,0,0,0,0,0,0,0,0]$} & {$[0,0,0,2,2,1,1,1,2]$} \\
34 & 0 & {$[0,0,0,0,0,0,0,0,0]$} & {$[0,0,0,0,1,2,1,2,2]$} \\
38 & 27 & {$[0,0,0,2,2,1,0,2,2]$} & {$[0,0,0,0,2,1,2,0,0]$} \\
41 & 0 & {$[0,0,0,0,0,0,0,0,0]$} & {$[0,0,0,0,2,2,0,0,0]$} \\
43 & 0 & {$[0,0,0,0,0,0,0,0,0]$} & {$[0,0,0,2,0,1,0,2,2]$} \\
47 & 0 & {$[0,0,0,0,0,0,0,0,0]$} & {$[0,0,0,2,0,2,2,2,2]$} \\
50 & 0 & {$[0,0,0,0,0,0,0,0,0]$} & {$[0,0,0,1,2,1,0,1,0]$} \\
52 & 27 & {$[0,0,0,2,0,0,2,0,0]$} & {$[0,0,0,0,2,1,1,2,2]$} \\
58 & 108 & {$[0,0,0,1,2,2,0,0,0]$} & {$[0,0,0,0,0,1,1,2,2]$} \\
59 & 0 & {$[0,0,0,0,0,0,0,0,0]$} & {$[0,0,0,2,1,1,0,1,0]$} \\
61 & 0 & {$[0,0,0,0,0,0,0,0,0]$} & {$[0,0,0,0,0,2,1,0,0]$} \\
67 & 0 & {$[0,0,0,0,0,0,0,0,0]$} & {$[0,0,0,0,0,2,2,0,0]$} \\
\hline & & &
\end{tabular}

Table 4: E33a1 with Weierstrass equation $y^{2}+x y=x^{3}+x^{2}-11 x$, which has non-split multiplicative reduction at $p=3$.

\begin{tabular}{cccc}
\hline$\Delta$ & $L^{*}$ & $\mathbf{1}\left(\mathbf{L}_{p}(E, \rho)\right)$ & $\mathbf{1}\left(\mathbf{L}_{p}(E, \sigma)\right)$ \\
\hline 2 & 2 & {$[2,0,0,0,0,0,0,0,0]$} & {$[2,0,0,0,0,0,0,0,0]$} \\
5 & 2 & {$[2,2,1,0,1,2,1,0,0]$} & {$[2,0,1,0,0,2,1,1,2]$} \\
7 & 2 & {$[1,2,1,2,0,1,0,2,2]$} & {$[1,1,1,1,0,1,2,0,0]$} \\
11 & 0 & {$[0,0,0,0,0,0,0,0,0]$} & {$[0,1,0,0,0,2,0,0,0]$} \\
13 & 50 & {$[1,2,1,2,2,0,2,2,2]$} & {$[1,2,2,0,2,0,2,2,2]$} \\
14 & 32 & {$[2,0,2,2,0,1,0,2,2]$} & {$[2,2,2,2,0,2,1,1,2]$} \\
20 & 98 & {$[1,2,2,1,2,1,0,1,0]$} & {$[1,1,2,0,0,1,0,0,0]$} \\
22 & 0 & {$[0,0,0,0,0,0,0,0,0]$} & {$[0,2,0,0,0,1,1,0,0]$} \\
23 & 2 & {$[2,2,2,1,0,2,2,0,0]$} & {$[2,1,0,2,2,1,0,2,2]$} \\
29 & 162 & {$[0,0,0,0,2,0,0,2,2]$} & {$[0,0,0,2,2,0,1,0,0]$} \\
31 & 32 & {$[1,2,1,2,1,2,0,0,0]$} & {$[1,0,2,1,2,0,1,0,0]$} \\
34 & 338 & {$[1,1,0,2,2,2,1,2,2]$} & {$[1,0,0,1,1,2,2,2,2]$} \\
38 & 8 & {$[2,2,2,0,0,1,2,0,0]$} & {$[2,2,2,0,2,0,1,2,2]$} \\
41 & 200 & {$[2,2,1,2,1,0,2,0,0]$} & {$[2,0,2,2,1,0,2,0,0]$} \\
\hline
\end{tabular}


Table 5: E15a1 with equation $y^{2}+x y+y=x^{3}+x^{2}-10 x-10$, which has split multiplicative reduction at $p=5$.

\begin{tabular}{cccc}
\hline$\Delta$ & $L^{*}$ & $\mathbf{1}\left(\mathbf{L}_{p}(E, \rho)\right)$ & $\frac{\log _{p}\left(q_{E, p}\right)}{\operatorname{ord}_{p}\left(q_{E, p}\right)} \mathcal{L}_{E}\left(\operatorname{Ind}_{K}^{\mathbb{Q}}(\mathbf{1})\right)$ \\
\hline 2 & 0 & {$[0,0,0,0,0,0,0,0,0]$} & {$[0,2,0,1,1,3,4,2,4]$} \\
3 & 80 & {$[0,1,4,0,1,2,4,3,4]$} & {$[0,0,2,4,1,4,1,4,4]$} \\
6 & 320 & {$[0,1,4,1,4,4,2,3,4]$} & {$[0,0,2,4,1,4,1,4,4]$} \\
9 & 80 & {$[0,1,4,0,1,2,4,3,4]$} & {$[0,0,2,4,1,4,1,4,4]$} \\
\hline
\end{tabular}

Table 6: E30a1 with Weierstrass equation $y^{2}+x y+y=x^{3}+x+2$, which has non-split multiplicative reduction at $p=5$.

\begin{tabular}{cccc}
\hline$\Delta$ & $L^{*}$ & $\mathbf{1}\left(\mathbf{L}_{p}(E, \rho)\right)$ & $\mathbf{1}\left(\mathbf{L}_{p}(E, \sigma)\right)$ \\
\hline 2 & 0 & {$[0,0,0,0,0,0,0,0,0]$} & {$[0,1,1,1,1,1,1,1,0]$} \\
3 & 0 & {$[0,0,0,0,0,0,0,0,0]$} & {$[0,2,0,1,4,0,3,2,4]$} \\
6 & 180 & {$[0,1,4,4,4,4,4,4,4]$} & {$[0,0,1,3,3,1,2,2,0]$} \\
9 & 0 & {$[0,0,0,0,0,0,0,0,0]$} & {$[0,2,0,1,4,0,3,2,4]$} \\
\hline
\end{tabular}

Table 7: E35a1 with Weierstrass equation $y^{2}+y=x^{3}+x^{2}+9 x+1$, which has non-split multiplicative reduction at $p=5$.

\begin{tabular}{cccc}
\hline$\Delta$ & $L^{*}$ & $\mathbf{1}\left(\mathbf{L}_{p}(E, \rho)\right)$ & $\mathbf{1}\left(\mathbf{L}_{p}(E, \sigma)\right)$ \\
\hline 2 & 0 & {$[0,0,0,0,0,0,0,0,0]$} & {$[0,0,0,0,0,0,0,0,0]$} \\
3 & 0 & {$[0,0,0,0,0,0,0,0,0]$} & {$[0,0,0,0,0,0,0,0,0]$} \\
\hline
\end{tabular}

Table 8: E55a1 with Weierstrass equation $y^{2}+x y=x^{3}-x^{2}-4 x+3$, which has split multiplicative reduction at $p=5$.

\begin{tabular}{cccc}
\hline$\Delta$ & $L^{*}$ & $\mathbf{1}\left(\mathbf{L}_{p}(E, \rho)\right)$ & $\frac{\log _{p}\left(q_{E, p}\right)}{\operatorname{ord}_{p}\left(q_{E, p}\right)} \mathcal{L}_{E}\left(\operatorname{Ind}_{K}^{\mathbb{Q}}(\mathbf{1})\right)$ \\
\hline 2 & 20 & {$[0,4,0,0,0,0,0,0,0]$} & {$[0,0,0,0,0,0,0,0,0]$} \\
\hline
\end{tabular}

Table 9: E70a1 with equation $y^{2}+x y+y=x^{3}-x^{2}+2 x-3$, which has non-split multiplicative reduction at $p=5$.

\begin{tabular}{cccc}
\hline$\Delta$ & $L^{*}$ & $\mathbf{1}\left(\mathbf{L}_{p}(E, \rho)\right)$ & $\mathbf{1}\left(\mathbf{L}_{p}(E, \sigma)\right)$ \\
\hline 2 & 0 & {$[0,0,0,0,0,0,0,0,0]$} & {$[0,3,3,3,3,3,3,3,4]$} \\
\hline
\end{tabular}

